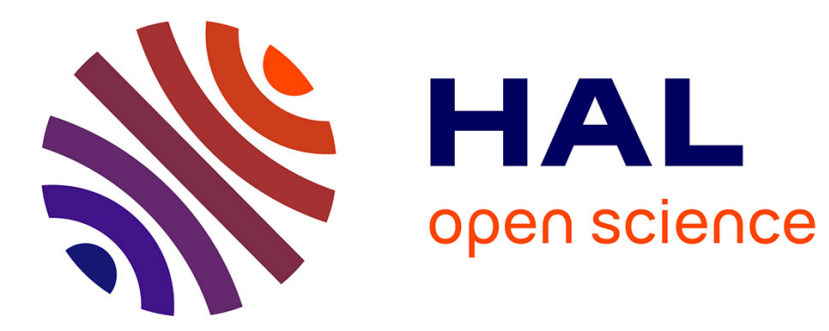

\title{
2FHL: The Second Catalog of Hard Fermi -LAT Sources
}

M. Ackermann, M. Ajello, William B. Atwood, L. Baldini, J. Ballet, Guido

Barbiellini, D. Bastieri, J. Becerra Gonzalez, R. Bellazzini, E. Bissaldi, et al.

\section{To cite this version:}

M. Ackermann, M. Ajello, William B. Atwood, L. Baldini, J. Ballet, et al.. 2FHL: The Second Catalog of Hard Fermi -LAT Sources. The Astrophysical Journal Supplement, 2016, 222 (1), 10.3847/00670049/222/1/5 . insu-01360302

\section{HAL Id: insu-01360302 \\ https://hal-insu.archives-ouvertes.fr/insu-01360302}

Submitted on 14 Sep 2018

HAL is a multi-disciplinary open access archive for the deposit and dissemination of scientific research documents, whether they are published or not. The documents may come from teaching and research institutions in France or abroad, or from public or private research centers.
L'archive ouverte pluridisciplinaire HAL, est destinée au dépôt et à la diffusion de documents scientifiques de niveau recherche, publiés ou non, émanant des établissements d'enseignement et de recherche français ou étrangers, des laboratoires publics ou privés. 


\section{FHL: THE SECOND CATALOG OF HARD FERMI-LAT SOURCES}

M. Ackermann ${ }^{1}$, M. Ajello ${ }^{2}$, W. B. Atwood ${ }^{3}$, L. Baldini ${ }^{4,5}$, J. Ballet ${ }^{6}$, G. Barbiellini ${ }^{7,8}$, D. Bastieri ${ }^{9,10}$, J. Becerra Gonzalez ${ }^{11,12}$, R. Bellazzini ${ }^{13}$, E. Bissaldi ${ }^{14}$, R. D. Blandford ${ }^{5}$, E. D. Bloom ${ }^{5}$, R. Bonino ${ }^{15,16}$, E. BotTacini ${ }^{5}$, T. J. BrandT ${ }^{11}$, J. Bregeon ${ }^{17}$, P. Bruel ${ }^{18}$, R. Buehler ${ }^{1}$, S. Buson ${ }^{11,19,20}$, G. A. Caliandro ${ }^{5,21}$, R. A. Cameron ${ }^{5}$, R. Caputo ${ }^{3}$, M. Caragiulo ${ }^{14,22}$, P. A. Caraveo ${ }^{23}$, E. Cavazzuti ${ }^{24}$, C. Cecchi $^{25,26}$, E. Charles $^{5}$, A. Chekhtman ${ }^{27}$, C. C. Cheung ${ }^{28}$,

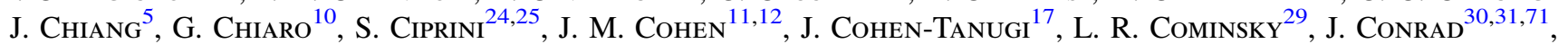
A. Cuoco ${ }^{15,16}$, S. Cutini ${ }^{24,25,32}$, F. D’Ammando ${ }^{33,34}$, A. De Angelis ${ }^{35}$, F. De Palma ${ }^{14,36}$, R. Desiante ${ }^{15,37}$, M. Di Mauro ${ }^{5}$, L. Di Venere ${ }^{14,22}$, A. Domínguez ${ }^{2}$, P. S. Drell ${ }^{5}$, C. Favuzzi ${ }^{14,22}$, S. J. Fegan ${ }^{18}$, E. C. Ferrara ${ }^{11}$, W. B. Focke ${ }^{5}$, P. Fortin ${ }^{38}$, A. FranckowiaK ${ }^{5}$, Y. Fukazawa ${ }^{39}$, S. FunK ${ }^{40}$, A. K. Furniss ${ }^{5}$, P. Fusco ${ }^{14,22}$, F. Gargano ${ }^{14}$, D. Gasparrini ${ }^{24,25}$, N. Giglietto ${ }^{14,22}$, P. Giommi ${ }^{24}$, F. Giordano ${ }^{14,22}$, M. Giroletti ${ }^{33}$, T. Glanzman ${ }^{5}$, G. Godfrey ${ }^{5}$, I. A. Grenier ${ }^{6}$,

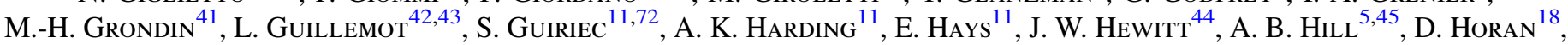
G. IAfrate ${ }^{7,46}$, Dieter HartmanN ${ }^{2}$, T. Jogler ${ }^{5}$, G. Jóhannesson ${ }^{47}$, A. S. Johnson ${ }^{5}$, T. Kamae ${ }^{48}$, J. Kataoka ${ }^{49}$,

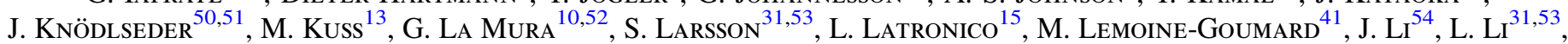

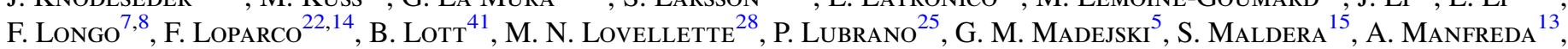
M. Mayer ${ }^{1}$, M. N. Mazziotta ${ }^{14}$, P. F. Michelson ${ }^{5}$, N. Mirabal ${ }^{11,73}$, W. Mitthumsiri ${ }^{55}$, T. Mizuno ${ }^{56}$, A. A. Moiseev ${ }^{12,20}$, M. E. Monzani ${ }^{5}$, A. Morsellis ${ }^{57}$, I. V. Moskalenko ${ }^{5}$, S. Murgia ${ }^{58}$, E. Nuss ${ }^{17}$, T. Ohsugi ${ }^{56}$, N. Omodei ${ }^{5}$, M. Orienti ${ }^{33}$, E. Orlando ${ }^{5}$, J. F. Ormes ${ }^{59}$, D. Paneque ${ }^{5,60}$, J. S. Perkins ${ }^{11}$, M. Pesce-Rollins ${ }^{5,13}$, V. Petrosian ${ }^{5}$, F. Piron ${ }^{17}$, G. Pivato ${ }^{13}$, T. A. Porter ${ }^{5}$, S. Rainò ${ }^{14,22}$, R. Rando ${ }^{9,10}$, M. Razzano ${ }^{13,73}$, S. Razzaque ${ }^{61}$, A. Reimer ${ }^{5,52}$, O. Reimer ${ }^{5,52}$, T. Reposeur ${ }^{41}$, R. W. Romani ${ }^{5}$, M. SÁnchez-Conde ${ }^{30,31}$, P. M. SAz Parkinson ${ }^{3,62}$, J. Schmid ${ }^{6}$, A. Schulz ${ }^{1}$, C. SGrò ${ }^{13}$, E. J. Siskind ${ }^{63}$,

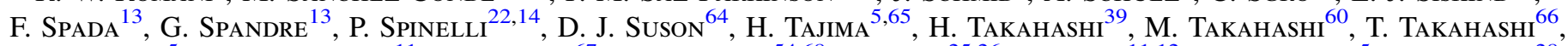
J. B. Thayer ${ }^{5}$, D. J. Thompson ${ }^{11}$, L. Tibaldo ${ }^{67}$, D. F. Torres ${ }^{54,68}$, G. Tosti ${ }^{25,26}$, E. Troja ${ }^{11,12}$, G. Vianello ${ }^{5}$, K. S. Wood ${ }^{28}$, M. Wood ${ }^{5}$, M. Yassine ${ }^{17}$, G. ZahariJas ${ }^{69,70}$, and S. Zimmer ${ }^{30,31}$

${ }^{1}$ Deutsches Elektronen Synchrotron DESY, D-15738 Zeuthen, Germany

${ }^{2}$ Department of Physics and Astronomy, Clemson University, Kinard Lab of Physics,

Clemson, SC 29634-0978, USA; majello@g.clemson.edu, alberto@clemson.edu

${ }^{3}$ Santa Cruz Institute for Particle Physics, Department of Physics and Department of Astronomy and Astrophysics, University of California at Santa Cruz, Santa Cruz, CA 95064, USA

${ }^{4}$ Università di Pisa and Istituto Nazionale di Fisica Nucleare, Sezione di Pisa I-56127 Pisa, Italy

${ }^{5}$ W. W. Hansen Experimental Physics Laboratory, Kavli Institute for Particle Astrophysics and Cosmology, Department of Physics and SLAC National Accelerator Laboratory, Stanford University, Stanford, CA 94305, USA

${ }^{6}$ Laboratoire AIM, CEA-IRFU/CNRS/Université Paris Diderot, Service d'Astrophysique, CEA Saclay, F-91191 Gif sur Yvette, France

${ }^{7}$ Istituto Nazionale di Fisica Nucleare, Sezione di Trieste, I-34127 Trieste, Italy ${ }^{8}$ Dipartimento di Fisica, Università di Trieste, I-34127 Trieste, Italy

${ }^{9}$ Istituto Nazionale di Fisica Nucleare, Sezione di Padova, I-35131 Padova, Italy

${ }^{10}$ Dipartimento di Fisica e Astronomia “G. Galilei,”Università di Padova, I-35131 Padova, Italy

${ }^{11}$ NASA Goddard Space Flight Center, Greenbelt, MD 20771, USA; jcohen@astro.umd.edu

${ }^{12}$ Department of Physics and Department of Astronomy, University of Maryland, College Park, MD 20742, USA

${ }_{14}^{13}$ Istituto Nazionale di Fisica Nucleare, Sezione di Pisa, I-56127 Pisa, Italy

${ }^{14}$ Istituto Nazionale di Fisica Nucleare, Sezione di Bari, I-70126 Bari, Italy

${ }^{15}$ Istituto Nazionale di Fisica Nucleare, Sezione di Torino, I-10125 Torino, Italy

${ }_{17}^{16}$ Dipartimento di Fisica Generale “Amadeo Avogadro,”Università degli Studi di Torino, I-10125 Torino, Italy

${ }^{17}$ Laboratoire Univers et Particules de Montpellier, Université Montpellier, CNRS/IN2P3, Montpellier, France

${ }^{19}$ Department of Physics and Center for Space Sciences and Technology, University of Maryland Baltimore County, Baltimore, MD 21250 , USA

${ }^{20}$ Center for Research and Exploration in Space Science and Technology (CRESST) and NASA Goddard Space Flight Center, Greenbelt, MD 20771, USA

22 Dipartimento di Fisica "M. Merlin" dell’Università e del Politecnico di Bari, I-70126 Bari, Italy

${ }^{23}$ INAF-Istituto di Astrofisica Spaziale e Fisica Cosmica, I-20133 Milano, Italy

24 Agenzia Spaziale Italiana (ASI) Science Data Center, I-00133 Roma, Italy; sarac@slac.stanford.edu, gasparrini@asdc.asi.it

${ }_{25}^{25}$ Istituto Nazionale di Fisica Nucleare, Sezione di Perugia, I-06123 Perugia, Italy

${ }^{26}$ Dipartimento di Fisica, Università degli Studi di Perugia, I-06123 Perugia, Italy

${ }^{27}$ College of Science, George Mason University, Fairfax, VA 22030, USA
${ }^{2}$ Space Science Division, Naval Research Laboratory, Washington, DC 20375-5352, USA

${ }^{29}$ Department of Physics and Astronomy, Sonoma State University, Rohnert Park, CA 94928-3609, USA

${ }^{30}$ Department of Physics, Stockholm University, AlbaNova, SE-106 91 Stockholm, Sweden

31 The Oskar Klein Centre for Cosmoparticle Physics, AlbaNova, SE-106 91 Stockholm, Sweden

${ }^{2}$ INAF Osservatorio Astronomico di Roma, I-00040 Monte Porzio Catone (Roma), Italy

${ }^{33}$ INAF Istituto di Radioastronomia, I-40129 Bologna, Italy

${ }^{34}$ Dipartimento di Astronomia, Università di Bologna, I-40127 Bologna, Italy

${ }^{35}$ Dipartimento di Fisica, Università di Udine and Istituto Nazionale di Fisica Nucleare, Sezione di Trieste, Gruppo Collegato di Udine, I-33100 Udine, Italy

${ }^{36}$ Università Telematica Pegaso, Piazza Trieste e Trento, 48, I-80132 Napoli, Italy

${ }^{37}$ Università di Udine, I-33100 Udine, Italy

${ }^{38}$ Harvard-Smithsonian Center for Astrophysics, Cambridge, MA 02138, USA

${ }^{39}$ Department of Physical Sciences, Hiroshima University, Higashi-Hiroshima, Hiroshima 739-8526, Japan

${ }^{40}$ Erlangen Centre for Astroparticle Physics, D-91058 Erlangen, Germany

${ }^{41}$ Centre d'Études Nucléaires de Bordeaux Gradignan, IN2P3/CNRS, Université Bordeaux 1, BP120, F-33175 Gradignan Cedex, France 


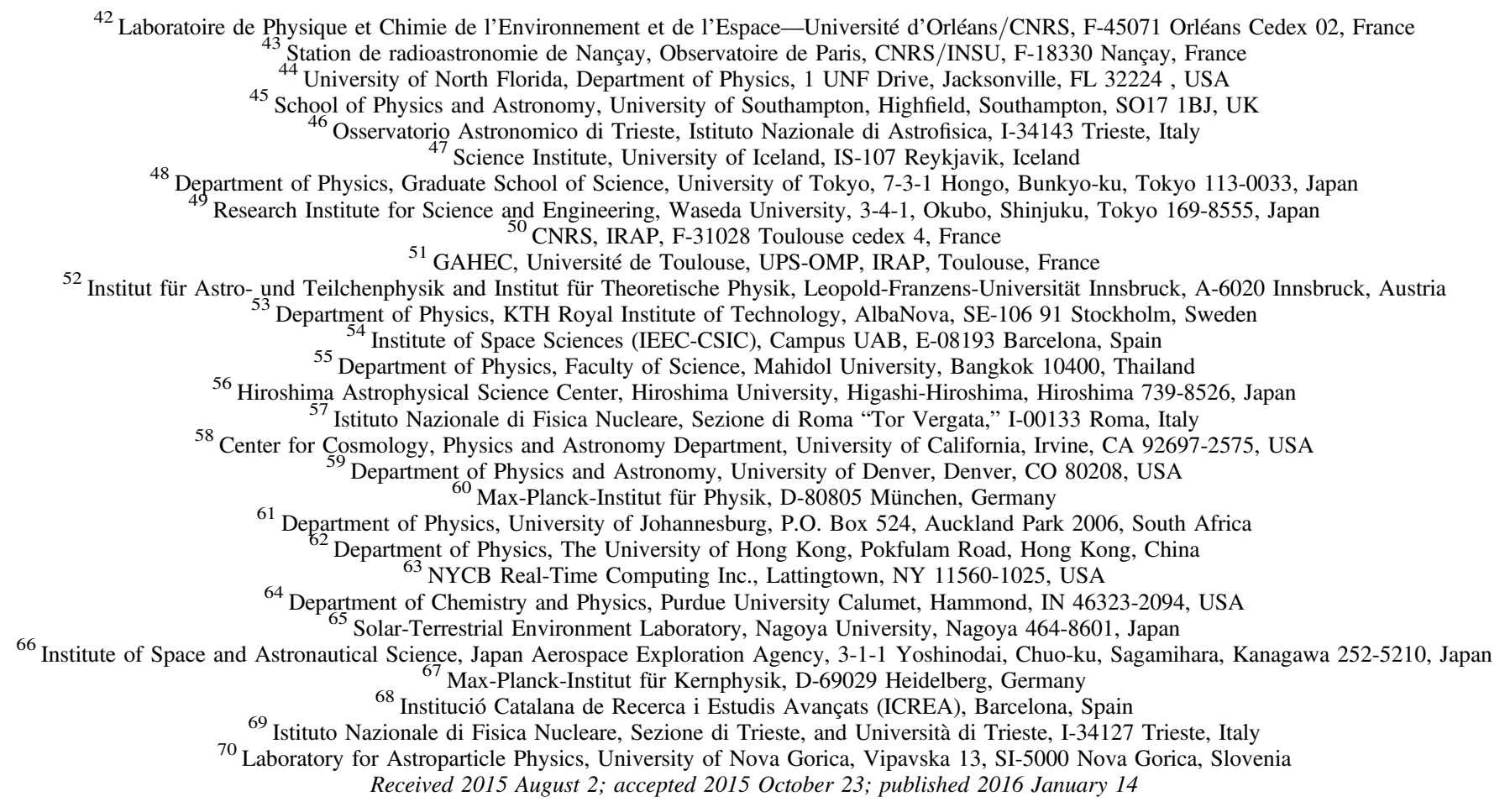

\section{ABSTRACT}

We present a catalog of sources detected above $50 \mathrm{GeV}$ by the Fermi-Large Area Telescope (LAT) in 80 months of data. The newly delivered Pass 8 event-level analysis allows the detection and characterization of sources in the $50 \mathrm{GeV}-2 \mathrm{TeV}$ energy range. In this energy band, Fermi-LAT has detected 360 sources, which constitute the second catalog of hard Fermi-LAT sources (2FHL). The improved angular resolution enables the precise localization of point sources $(\sim 1 ! 7$ radius at $68 \%$ C. L.) and the detection and characterization of spatially extended sources. We find that $86 \%$ of the sources can be associated with counterparts at other wavelengths, of which the majority (75\%) are active galactic nuclei and the rest (11\%) are Galactic sources. Only 25\% of the 2FHL sources have been previously detected by Cherenkov telescopes, implying that the 2FHL provides a reservoir of candidates to be followed up at very high energies. This work closes the energy gap between the observations performed at $\mathrm{GeV}$ energies by Fermi-LAT on orbit and the observations performed at higher energies by Cherenkov telescopes from the ground.

Key words: catalogs - gamma-rays: general

Supporting material: FITS files

\section{INTRODUCTION}

The Large Area Telescope (LAT, Atwood et al. 2009) on board the Fermi gamma-ray space telescope has been surveying the whole sky since 2008 August. Its unprecedented sensitivity and localization accuracy allowed the detection of over 3000 point-like sources in 4 years of data (see the third catalog of Fermi-LAT sources, 3FGL, Acero et al. 2015). Typically, Fermi-LAT catalog studies are based on source detection and characterization in the whole $0.1-100 \mathrm{GeV}$ energy band. The larger photon statistics present at low energy, counterbalanced by the LAT point-spread function (PSF) whose size decreases with energy, yields an optimum sensitivity at few-GeV energies. The Fermi-LAT catalogs are thus representative of the GeV sky more than they are of the $\mathrm{MeV}$ or the sub-TeV sky.

\footnotetext{
71 Wallenberg Academy Fellow.

72 NASA Postdoctoral Program Fellow, USA.

73 Funded by contract FIRB-2012-RBFR12PM1F from the Italian Ministry of Education, University and Research (MIUR).
}

The first Fermi-LAT catalog of hard sources, named 1FHL (Ackermann et al. 2013), provided an unbiased census of the sky at energies from 10 up to $500 \mathrm{GeV}$. The comparison of 1FHL and $0.1-100 \mathrm{GeV}$ observations (as provided in Nolan et al. 2012) allowed us to uncover the presence of spectral breaks and to determine that blazars of the BL Lacertae (BL Lac) type represented about $50 \%$ of the entire source population detected in that band. All-sky surveys at $\gamma$-ray energies are instrumental for ground-based imaging atmospheric Cherenkov telescopes (IACTs) such as H.E.S.S., MAGIC, and VERITAS (Holder et al. 2009; Lorenz 2004; Hinton 2004, respectively) in order to find new sources because of their limited fields of view.

Recently, a new event-level analysis (known as Pass 8) has been developed by the Fermi-LAT collaboration (Atwood et al. 2013a, 2013b). Pass 8 significantly improves the LAT's background rejection, PSF, and effective area. All these enhancements lead to a significant increase of the LAT sensitivity and its effective energy range, from below $100 \mathrm{MeV}$ to beyond a few hundred GeV (Atwood et al. 

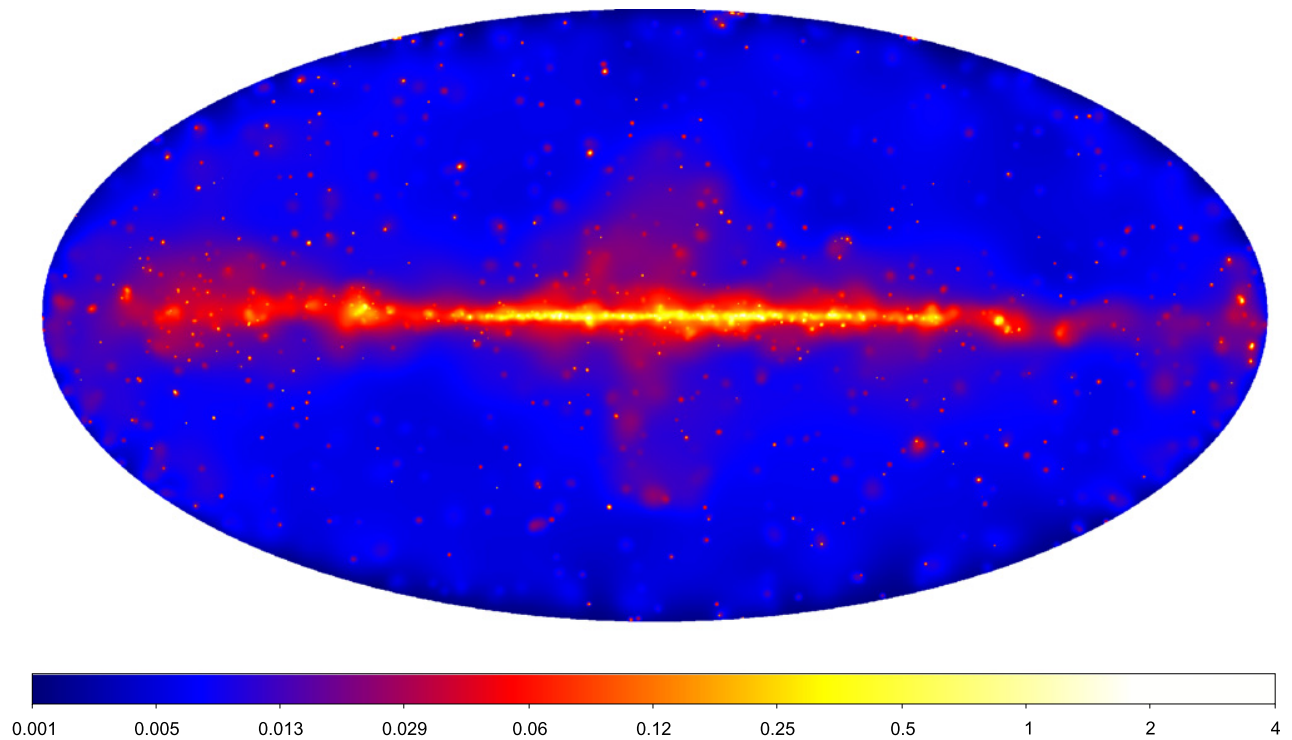

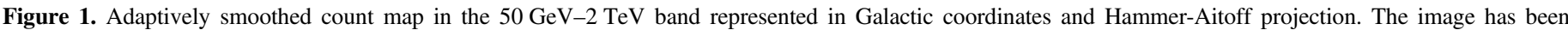

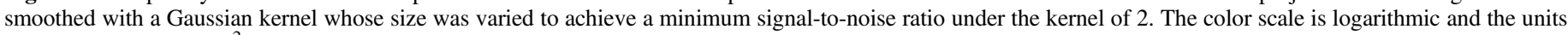
are counts per $(0.1 \mathrm{deg})^{2}$.

2013a, 2013b). These improvements are particularly significant above $50 \mathrm{GeV}$, yielding an enhancement in the acceptance and PSF by a factor between 1.2 and 2. It is interesting to note that, above $50 \mathrm{GeV}$, both the PSF (governed mostly by the pitch of the tracker silicon strips and the spacing of the tracker planes) and the effective area of the LAT are only weakly dependent on energy and that the LAT operates, due to the (almost complete) absence of background, in the photon-limited regime.

In this paper we use 80 months of Pass 8 data to produce a catalog of sources detected by the LAT at energies ${ }^{74}$ between $50 \mathrm{GeV}$ and $2 \mathrm{TeV}$. This constitutes the second catalog of hard Fermi-LAT sources, named 2FHL, which allows a thorough study of the properties of the whole sky in the sub-TeV domain.

The paper is organized as follows: Section 2 describes the analysis, and Section 3 discusses the 2FHL catalog and the main results. A summary is provided in Section 4.

\section{ANALYSIS}

\subsection{Data Selection}

We use 80 months (from 2008 August to 2015 April) of P8_SOURCE photons with reconstructed energy in the $50 \mathrm{GeV}-2 \mathrm{TeV}$ range. At these energies the LAT has an energy resolution of around $10 \%-15 \%(1 \sigma)$. Photons detected at zenith angles larger than $105^{\circ}$ were excised to limit the contamination from $\gamma$-rays generated by cosmic-ray interactions in the upper layers of the atmosphere. Moreover, data were filtered removing time periods when the instrument was not in sky-survey mode. ${ }^{75}$ This leaves approximately 61,000 photons detected all over the sky. The count map reported in Figure 1 shows that Fermi-LAT observes many point-like sources and large-scale diffuse emission in the direction of our Galaxy, some of which appears coincident with the so-called Fermi bubbles (Su et al. 2010; Ackermann et al. 2014b).

\footnotetext{
${ }_{75}$ Note the different energy range with respect to the 1FHL.

75 This was achieved using the expression "(DATA_QUAL $>0) \& \&$ (LAT_CONFIG ==1)" in gtmktime.
}

\subsection{Source Detection}

The first step of the source detection stage comprises the identification of source seeds, which are locations of potential sources whose significance is later tested through a maximum likelihood (ML) analysis. Seeds are identified via a sliding-cell algorithm as excesses above the background, as clusters of 3 or more photons, and via a wavelet analysis (Ciprini et al. 2007). Moreover, the seed list includes all the point sources detected in the 1FHL catalog. We note that this seed list may include statistical fluctuations as well as real sources with a nonoptimal position.

A full ML analysis is then performed in order to verify which, among the seeds, are the reliable sources. The analysis is performed in 154 regions of interest (ROIs), varying between $10^{\circ}$ and $20^{\circ}$ in radius, whose sizes and positions in the sky are optimized to cover all the seeds, ensuring that no more than 45 seeds are contained in a single ROI. For each ROI, we build a sky model that includes all the potential sources in the region as well as the Galactic and isotropic diffuse emissions. ${ }^{76}$ These models, which are defined only up to $\sim 600 \mathrm{GeV}$ and $\sim 900 \mathrm{GeV}$, respectively, where extrapolated up to $2 \mathrm{TeV}$. The ROI models include also the extended sources present in the region (see Section 2.3). The model is fit to the data via the unbinned ML algorithm provided within the Fermi Science Tools $^{77}$ (version v9r34p3).

The spectrum of each source is modeled with a power law because none of the sources is expected to show statistically significant spectral curvature detectable by the LAT in this energy band. Indeed, this was the case for the sources in the 1FHL catalog (Ackermann et al. 2013).

The fit is performed iteratively in order to ensure convergence and to produce an optimal solution. It proceeds as follows:

\footnotetext{
$\overline{76}$ We use the gll_iem_v06.fits and iso_P8R2_SOURCE_V6_v06. txt templates available at http://fermi.gsfc.nasa.gov/ssc/data/access/lat/ BackgroundModels.html

77 Available at http://fermi.gsfc.nasa.gov/ssc/data/analysis/software/
} 
1. Complex ML fits require approximate knowledge of the starting values of the parameters. For this reason the first step aims to find those values by fitting each single source separately to determine approximate spectral parameters. Throughout the entire process, the parameters of the diffuse emission models are left free to vary. The significance of each source is evaluated using the test statistic TS $=2\left(\ln \mathcal{L}_{1}-\ln \mathcal{L}_{0}\right)$, where $\mathcal{L}_{0}$ and $\mathcal{L}_{1}$ are the likelihoods of the background (null hypothesis) and the hypothesis being tested (e.g., source plus background). At each step in the procedure, marginal sources, those with TS $<10$, are removed from the model. Once the spectral parameters and significance of each source have been evaluated, a global fit for which all the parameters of the sources with a $\mathrm{TS} \geqslant 10$ are allowed to vary is performed. Then one more global fit is performed after removing all the sources that had $\mathrm{TS}<10$ at the previous global fit. This step, as well as all the others, includes sources that are spatially extended (see Section 2.3);

2 . In this second step, the positions of point-like sources, using the best-fit sky model derived at step 1, are optimized using the gtfindsrc tool. This step is done iteratively as well by optimizing first the positions of the most significant sources found at step 1 and later those of the fainter ones;

3. The parameters and significances of sources are estimated again (as in step 1) using the best-fit source positions. This step produces the best-fit sky model for any given ROI. Seeds with $10 \leqslant$ TS $<25$ are included in the model, but not reported in the final catalog;

4. For each source we estimate the energy of the highestenergy photon (HEP) that the fit attributes robustly to the source model. This is done using the tool gtsrcprob and selecting the HEP that has a probability $>85 \%$ to belong to the source;

5. A spectrum with three logarithmically spaced bins (boundaries of $50 \mathrm{GeV}, 171 \mathrm{GeV}, 585 \mathrm{GeV}, 2 \mathrm{TeV}$ ) is generated for each source in the ROI that is detected with TS $\geqslant 25$ and with the number of detected $\gamma$ rays (estimated by the likelihood, $N_{\text {pred }}$ ) to be $\geqslant 3$.

The procedure described above achieves the detection of 360 sources (including the extended sources discussed next at Section 2.3) with TS $\geqslant 25$ and $N_{\text {pred }} \geqslant 3$ across the entire sky. The number of seeds kept in the ROI models with $10 \leqslant \mathrm{TS}<25$ is 453 , while 7 are the seeds with TS $\geqslant 25$, but $N_{\text {pred }}<3$. We have performed seven Monte Carlo simulations of the $>50 \mathrm{GeV}$ sky whose data have been analyzed like the real data (as detailed above). The $N_{\text {pred }}$ cut was introduced on the basis of these simulations to limit to $\lesssim 1 \%$ the number of false positives in the final catalog. These simulations will be discussed in a forthcoming publication.

\subsection{Search for Spatially Extended Sources}

Preliminary runs of the source detection method described in Section 2.2 detected clusters of point sources in the Galactic plane, which were suggestive of spatially extended sources. It is also possible that clusters of seed sources, each with subdetection-threshold significance, could be detected as a significant extended source. Not modeling extended $\gamma$-ray emission as such can lead to inaccurate measurements of spectral and spatial properties of both the extended source and neighboring point sources, particularly in the Galactic plane (Lande et al. 2012). Most of the TeV sources in the Galactic plane are spatially extended (Carrigan et al. 2013; Ong 2013), so to clearly connect LAT detections spectrally to these sources, extension detection and characterization is important. In the following, we distinguish between sources whose extension has been previously determined with Fermi-LAT and new extended sources that are reported for the first time in a Fermi-LAT catalog. The details of all significantly detected extended sources will be reported in Section 3.3.2.

\subsubsection{Extended Sources Previously Detected by the LAT}

We explicitly modeled sources as spatially extended when a previous, dedicated, analysis found the source to be resolved by the LAT. The 25 extended sources reported in 3FGL were included in our model using the spatial templates derived in the individual source studies (see references in Acero et al. 2015). Refitting the positions and extensions of the 3FGL extended sources in this energy range is beyond the scope of this work.

Of the 25 3FGL extended sources, 19 are significantly detected here above the detection threshold ( $T S \geqslant 25$ ). Only 6 sources are not detected and, since all have $\mathrm{TS}<10$, are removed from the sky model (see Section 3.3.2 for details).

One extended LAT source has had a dedicated analysis published since the release of the 3FGL catalog. Abramowski et al. (2015a) reported joint H.E.S.S. and LAT observations of the very high energy (VHE) source HESS J1834-087. This source is coincident with supernova remnant (SNR) W41 and was detected as spatially extended in a wide energy range spanning $1.8 \mathrm{GeV}$ to $30 \mathrm{TeV}$. In this paper, we employ the spatial model for the GeV emission determined in Abramowski et al. (2015a), leading to a significant detection of this source.

\subsubsection{Newly Detected Extended Sources}

In addition to modeling the extended sources mentioned in Section 2.3.1, we performed a blind search of the Galactic plane $\left(|b|<10^{\circ}\right)$ to identify potential extended sources not included in previously published works. Our analysis pipeline is similar to that used in Hewitt et al. (2013), with some modifications tailored to searching for multiple extended sources in an ROI. The pipeline employs the pointlike binned ML package (Kerr 2010), in particular utilizing the extended source fitting tools validated by Lande et al. (2012) to simultaneously fit the position, extension, and spectra of sources in our ROI.

We created 72 ROIs of radius $10^{\circ}$, centered on $b=0^{\circ}$ with neighboring ROIs overlapping and separated by $5^{\circ}$ in Galactic longitude. Our initial model of the $\gamma$-ray emission in each ROI consisted solely of the Galactic diffuse (allowing just the normalization to be fit) and isotropic emission models (fixing the normalization), with no other sources in the ROI. Emission in the ROIs was further characterized by adding sources and fitting their spectral parameters (normalization and spectral index) in a $14^{\circ} \times 14^{\circ}$ region.

A TS map, that included all significant sources found previously, made up of $0.1 \times 0^{\circ} .1$ bins across the ROI, was created at each iteration and a small radius $\left(0^{\circ} .1\right)$ uniform disk, with a power-law spectrum was placed at the position of the peak TS pixel. The spectra of any newly added sources, as well as the position, extension, and spectral parameters of the disk were then fit. If $\mathrm{TS}_{\text {ext }} \geqslant 16$, where $\mathrm{TS}_{\text {ext }}=2 \log \left(\mathcal{L}_{\text {ext }} / \mathcal{L}_{\mathrm{ps}}\right)$ 
(i.e., twice the logarithm likelihood ratio of an extended to a point source, Lande et al. 2012), then the disk was kept in the model. For $\mathrm{TS}_{\mathrm{ext}}<16$, the extended source was replaced by a point source with a power-law spectral model. For the pointsource replacement case, spectral parameters of sources in the ROI were fit and the position of the new point source was optimized. Finally, the spatial parameters of any previously added extended sources were refit iteratively before creating a new TS map and repeating the process. We stopped adding sources when the peak TS was less than 16 for two successive sources.

To assess the impact of fitting extended sources when starting with an ROI devoid of sources, a crosscheck analysis (also using pointlike) was performed across the Galactic plane. We included 3FGL point and extended sources, the Galactic diffuse and isotropic emission, and pulsars from the second Fermi-LAT pulsar catalog (Abdo et al. 2013; as well as from 3FGL) in the preliminary source model for each region. Sources were iteratively added to account for residual emission and both these residual sources and 3FGL sources were tested for extension. Remarkably, this alternative analysis converges (i.e., spectral and spatial parameters for the detected extended sources are compatible in both analyses) to the initially sourcedevoid analysis for nearly all detected extended sources.

Extended sources detected in the analysis described in this section for which the position and extension were compatible with those found by the crosscheck were included in the ROI model at step 1 of the full ML analysis detailed in Section 2.2. Seed point sources interior to the extended sources were removed prior to the ML fit. To address the ambiguity between detecting a source as spatially extended as opposed to a combination of point sources, we utilized the algorithm detailed in Lande et al. (2012) to simultaneously fit the spectra and positions of two nearby point sources. We only consider a source to be extended if $\mathrm{TS}_{\text {ext }}>\mathrm{TS}_{2 \mathrm{pts}}$ (improvement when adding a second point source). Our blind search of the Galactic plane allowed us to find 5 sources not previously detected as extended by Fermi-LAT. Further details on these sources are presented in Section 3.3.2.

\subsection{Comparison with Pass 7}

In order to gauge the improvement delivered by Pass 8 at $\geqslant 50 \mathrm{GeV}$, we repeated the analysis procedure described above with 80 months of Pass 7 reprocessed data. This analysis detected $\sim 230$ sources; $\sim 35 \%$ fewer than the corresponding analysis that relies on Pass 8 data. The main difference is for the region $|b|>10^{\circ}$ where Pass 8 data, because of the larger acceptance, and better PSF allow the detection of $\sim 60 \%$ more sources than what could be achieved with Pass 7. The improvements delivered by Pass 8 above $50 \mathrm{GeV}$ are clearly substantial.

\subsection{Source Association}

The approach for automated source association closely follows that used for the 2FGL, 1FHL, 3FGL and 3LAC catalogs (Nolan et al. 2012; Ackermann et al. 2013; Acero et al. 2015; Ackermann et al. 2015). In short, we use catalogs of known or potential $\gamma$-ray source classes to determine the probability that a source from a given catalog or survey is associated with a 2 FHL source.
The associations were derived with two different procedures: the Bayesian method and the likelihood-ratio method (described in detail in Ackermann et al. 2015; Acero et al. 2015). In the application of these two methods, potential counterparts were deemed associated if they were found to have a posteriori probability of at least $80 \%$.

For the Bayesian method, the catalogs relavant for associating 2FHL sources are the 5th version of the BZCAT (Massaro et al. 2015) and the ATNF Pulsar Catalog (Manchester et al. 2005). Other catalogs of Galactic populations (X-ray binaries, O stars, Wolf-Rayet stars, luminous blue variable stars, open and globular clusters) were used in the procedure, but no counterparts reached the probability threshold.

For the likelihood-ratio association method, we made use of a number of relatively uniform radio surveys. Almost all radio candidates of possible interest are in the NRAO VLA Sky Survey (NVSS; Condon et al. 1998) or the Sydney University Molonglo Sky Survey (Mauch et al. 2003). To look for additional possible counterparts we cross-correlated the LAT sources with the most sensitive all-sky X-ray survey, the ROSAT All Sky Survey Bright and Faint Source Catalogs (Voges et al. 1999, 2000).

In order to be consistent, we evaluated matches between the 2FHL and the 3FGL and 1FHL catalogs, cross correlating those catalogs with the 2FHL one taking into account the source location uncertainty (95\% confidence) regions. In those cases, we adopted the source associations given in previously published Fermi-LAT catalogs. This yielded only two associations (Eta Carinae and the SNR G338.3-0.0), which were both detected and associated in both the 3FGL and 1FHL. The 2FHL catalog was also cross-correlated with the $\mathrm{TeVCat}^{78}$ (which contains all the TeV sources detected by IACTs so far) and spatial coincidences are reported (see Section 3.6). Since they have no positional error associated, the association probability was not computed for the extended sources.

High-confidence associations allow us to assess the systematic uncertainty in the accuracy of the LAT source positions. As done in Acero et al. (2015), we compared the distribution of angular separations of the $\gamma$-ray sources to the highest-confidence (probability $>90 \%$ ) counterparts (in units of $1 \sigma$ errors) with a Rayleigh distribution, and found it slightly broader than expected. Consequently, the standard $68 \%$ uncertainty radius provided by $g$ tfinds $\mathrm{r} \mathrm{C}$ has been multiplied by 1.08 and summed in quadrature to $0.003(68 \%$ absolute systematic error from $3 \mathrm{FGL}$ ).

\section{THE 2FHL CATALOG}

The 2FHL catalog includes 360 sources detected over the whole sky, each with a likelihood test statistic of TS $\geqslant 25$ and number of associated photons, $N_{\text {pred }} \geqslant 3$. The association procedure (see Section 2.5) finds that $75 \%$ of the sources in the catalog (274 sources) are extragalactic ${ }^{79}, 11 \%$ (38 sources) are of Galactic nature, and 13\% (48 sources) are unassociated (or associated with a $\mathrm{TeV}$ source of unknown nature). The unassociated sources are divided between 23 sources located at $|b|<10^{\circ}$, and 25 sources at $|b| \geqslant 10^{\circ}$. Therefore the fraction of extragalactic sources in the sample is likely larger than $80 \%$. The number of $2 \mathrm{FHL}$ sources that have not been reported in

\footnotetext{
78 See http://tevcat.uchicago.edu/

79 This includes N 157B, an extragalactic pulsar wind nebula (PWN).
} 
Table 1

2FHL Source Classes

\begin{tabular}{lcc}
\hline \hline Description & \multicolumn{2}{c}{ Associated } \\
\cline { 2 - 3 } & Designator & Number \\
\hline Pulsar & $\mathrm{psr}$ & 1 \\
Pulsar wind nebula & $\mathrm{pwn}$ & 14 \\
Supernova remnant & $\mathrm{snr}$ & 16 \\
Supernova remnant/Pulsar wind nebula & $\mathrm{spp}$ & 4 \\
High-mass binary & $\mathrm{hmb}$ & 2 \\
Binary & bin & 1 \\
Star-forming region & $\mathrm{sfr}$ & 1 \\
BL Lac type of blazar & bll & 180 \\
BL Lac type of blazar with prominent galaxy & bll-g & 13 \\
$\quad$ emission & & \\
FSRQ type of blazar & fsrq & 10 \\
Non-blazar active galaxy & agn & 2 \\
Radio galaxy & rdg & 4 \\
Radio galaxy/BL Lac & rdg/bll & 2 \\
Blazar candidate of uncertain type I & bcu I & 7 \\
Blazar candidate of uncertain type II & bcu II & 34 \\
Blazar candidate of uncertain type III & bcu III & 19 \\
Normal galaxy (or part) & gal & 1 \\
Galaxy cluster & galclu & 1 \\
Total associated & $\ldots$ & 312 \\
Unassociated & $\ldots$ & 48 \\
\hline Total in 2FHL & $\ldots$ & 360 \\
\hline
\end{tabular}

Note. The designation "spp" indicates potential association with SNR or PWN. The "bcu I," "bcu II," and "bcu III" classes are derived from 3LAC and describe the increasing lack of multiwavelength information to classify the source as a blazar (see Ackermann et al. 2015, for more details). The designation "bll-g" is adapted from the BZCAT (Massaro et al. 2015) and indicates a blazar whose SED has a significant contribution from the host galaxy.

3FGL is 57,47 of which have not been previously reported in any Fermi-LAT catalog nor in the TeVCat and are thus new $\gamma$ ray sources. The results of the association procedures are summarized in Table 1. Figure 2 shows the location of $2 \mathrm{FHL}$ sources color-coded according to their source class.

\subsection{Description of the Catalog}

The format of the 2FHL catalog follows that of previous Fermi-LAT catalogs (Ackermann et al. 2013; Acero et al. 2015) and it is detailed in Table 2, while an excerpt of the catalog itself, which will be fully released in FITS files ${ }^{80}$ format, is presented in Table 3. Table 3 contains 16 columns and it lists the 2FHL name, position, significance, spectral properties (along with their $1 \sigma$ uncertainties), association, class and redshift (if available) of the sources. The positions are given in degrees in both Equatorial (J2000) and Galactic coordinates with their $1 \sigma$ positional uncertainties (also in degrees). The full catalog, which contains 39 columns, also reports (as detailed in Table 2) energy fluxes in the whole $50 \mathrm{GeV}-2 \mathrm{TeV}$ energy band, and integrated photon fluxes in three logarithmically spaced energy bins together with their $1 \sigma$ uncertainties, and the number of photons attributed to the source $\left(N_{\text {pred }}\right)$. It also reports, for all objects, with redshift, the intrinsic (un-attenuated by the extragalactic background light; EBL) index with its $1 \sigma$ uncertainty, computed using the

\footnotetext{
${ }^{80}$ See: http://fits.gsfc.nasa.gov/
}

Domínguez et al. (2011b) and the Gilmore et al. (2012) EBL models (see Dominguez \& Ajello 2015 for details). Finally, the most likely association (with probability $>80 \%$ ) is also given with its probability. We also report the 3FGL, 1FHL, 1FGL, and TeVCat associations if any.

\subsection{General Characteristics of 2FHL Sources}

The 2FHL sources have $>50 \mathrm{GeV}$ fluxes ranging from $\sim 8 \times 10^{-12} \mathrm{ph} \mathrm{cm}^{-2} \mathrm{~s}^{-1}$ to $\sim 1.3 \times 10^{-9} \mathrm{ph} \mathrm{cm}^{-2} \mathrm{~s}^{-1}$ with a median flux of $2.0 \times 10^{-11} \mathrm{ph} \mathrm{cm}^{-2} \mathrm{~s}^{-1}$ and a median spectral index of 2.83. The index uncertainty increases rapidly with the spectral index (e.g., the uncertainty is about \pm 0.5 for sources with $\Gamma=2$ whereas it is \pm 2 for sources with $\Gamma=5$ ). Half of the sources are localized to better than 1!7 radius at $68 \%$ confidence. Figure 3 plots the spectral index versus the photon flux for sources associated with extragalactic sources or located at $|b| \geqslant 10^{\circ}$ (the extragalactic sample), Galactic sources, and unassociated sources. Figure 3 shows that there is no visible dependence of the sensitivity (i.e., minimum detectable photon flux) on the spectral index. This is because the size of the Pass 8 PSF remains approximately constant above $50 \mathrm{GeV}$. However, extragalactic sources are detected to lower fluxes than Galactic objects, highlighting that the sensitivity for source detection becomes worse in the plane of the Galaxy.

The distributions of spectral indices and the highest photon energy reported in Figure 4 show that extragalactic sources tend to have larger photon indices (median of 3.13) than Galactic sources (median of 2.10). Because of the harder spectra, Galactic sources tend to have higher-energy HEPs than those of extragalactic sources as shown as well in Figure 4. It is interesting to note that unassociated sources have a median index of 2.22 (2.00 for sources at $|b|<10^{\circ}$ and 2.96 for those at $|b| \geqslant 10^{\circ}$ ), showing that a fraction (see later) of unassociated sources is likely of Galactic origin.

Building a spectral energy distribution (SED) represents a powerful way to discriminate or infer the nature of a source. By combining the spectral data from the 3FGL, $1 \mathrm{FHL}$, and $2 \mathrm{FHL}$ catalogs, it becomes possible to measure the SEDs of sources over four decades in energy. Although these catalogs rely on different exposures and most $\gamma$-ray sources are variable, these data allow us to characterize the high-energy peak of their broadband SEDs. The SEDs of a few notable sources will be shown in the next sections.

\subsection{The 2FHL Galactic Source Population}

The narrow PSF core (about $0^{\circ} .1$ ) and moderate Galactic diffuse emission (in comparison with the $>100 \mathrm{MeV}$ band) allows the LAT to characterize and study well the emission of sources in the plane of our Galaxy above $50 \mathrm{GeV}$. Within $|b|<10^{\circ}$, Fermi-LAT has detected 103 sources. Of those, 38 sources are associated with Galactic sources, 42 to blazars, 14 are unassociated and 9 are associated with other $\gamma$-ray sources whose origin is not known (see below). Figure 5 shows cutouts of the Galactic plane with all detected sources labeled.

Among the 38 Galactic sources, 16 are spatially coincident with SNRs, 13 are coincident with PWNe, 4 are associated with $\mathrm{PWN} / \mathrm{SNR}$ complexes and the other 5 sources are X-ray binaries (3), one pulsar (PSR J0835-4510) and the Cygnus Cocoon. It is clear that the majority of Galactic sources detected above $50 \mathrm{GeV}$ are associated with objects at the final stage of stellar evolution. 


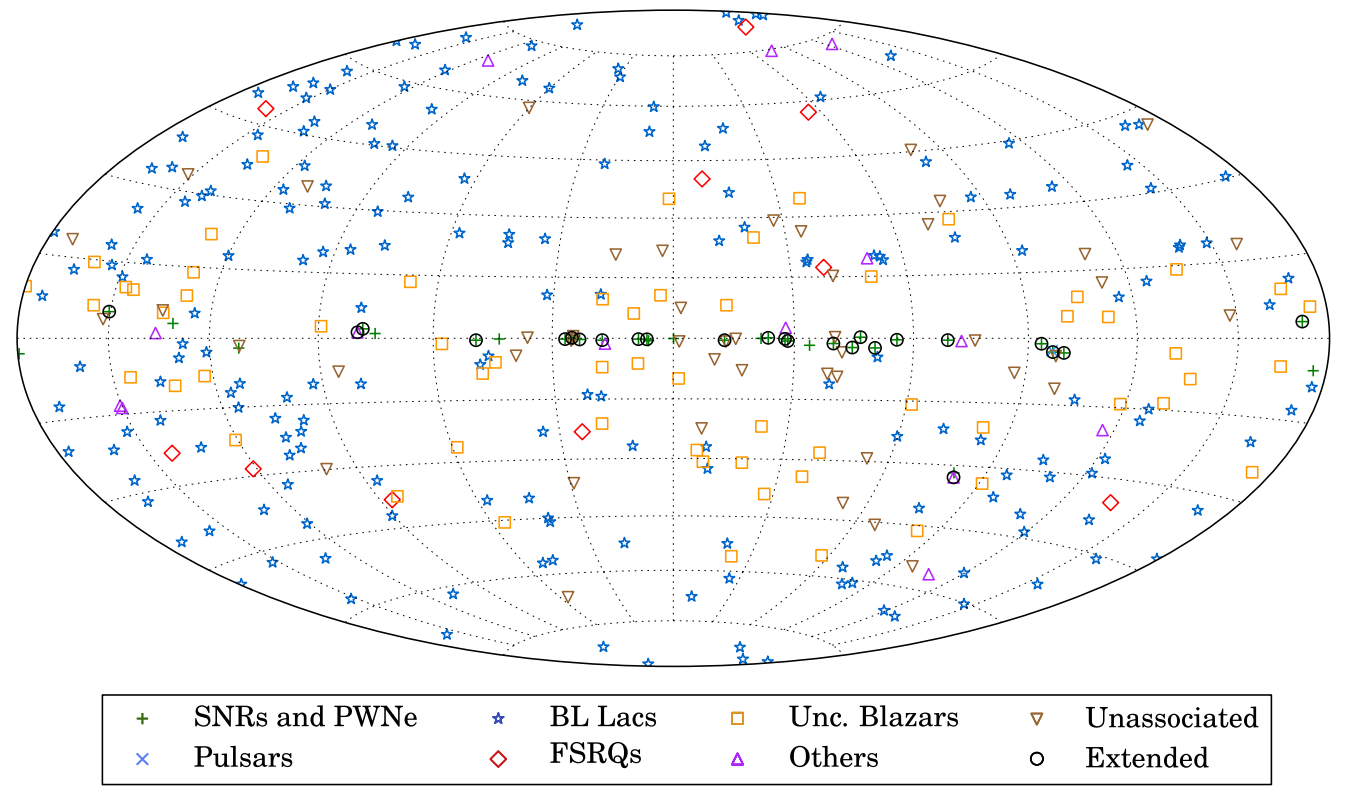

Figure 2. Sky map, in Galactic coordinates and Hammer-Aitoff projection, showing the sources in the 2FHL catalog classified by their most likely association.

Galactic sources display on average hard spectra, which is a sign of efficient particle acceleration. Roughly $55 \%$ of all Galactic sources have a spectral index lower than 2.2. For comparison, only $14 \%$ of the $2 \mathrm{FHL}$ blazars display such hard spectra. A sizable fraction (approximately 25\%, see Figure 4, left panel) of Galactic sources has a photon index harder than 2, implying a high-energy SED peak in the $\mathrm{TeV}$ band. Indeed, as the right panel of Figure 4 shows, Fermi-LAT detects emission from many Galactic sources well beyond $500 \mathrm{GeV}$. All PWNe detected by Fermi are found to be powered by young and energetic pulsars (age $\lesssim 30 \mathrm{kyr}$, Acero et al. 2013). While it is common for PWNe to show hard spectra, this is less so for SNRs whose majority (about $85 \%$ ) display softer spectra (Hewitt et al. 2013). Hard-spectrum SNRs are typically young or mid-aged $(\lesssim 3-5 \mathrm{kyr})$ and might be difficult to find in radio surveys. Thus, Galactic surveys at above $50 \mathrm{GeV}$ have the capability to detect new SNRs that might have been previously missed. Such an example is represented by the extended source 2 FHL J0431.2 +5553 e which is spatially coincident with a new SNR (SNR G150.3+4.5) recently reported by Gao \& Han (2014).

Of the 14 sources at $|b|<10^{\circ}$ that do not have an association, 7 have power-law indices harder than 2 which renders them likely Galactic objects. It is interesting to note that 6 of these 7 objects are offset from the plane of the Galaxy by more than $4^{\circ}$. This is in marked contrast with the associated portion of the sample where only the Crab Nebula and the newly discovered SNR G150.3+4.5 (out of 34 SNR/PWN systems) have such a large offset. Thus it seems unlikely that all these unassociated sources are SNR/PWN systems.

\subsubsection{Comparison with the H.E.S.S. Galactic Plane Survey}

The H.E.S.S array, with a field of view of about $5^{\circ}$ and an angular resolution of approximately 0.12 , has invested $2800 \mathrm{hr}$ of exposure to survey part ${ }^{81}$ of the Galactic plane, reaching an average sensitivity of $2 \%$ of the Crab Nebula flux (i.e.,

\footnotetext{
$\overline{81}$ The H.E.S.S. Galactic plane survey extends between $283^{\circ}<l<59^{\circ}$ and Galactic latitudes of $|b|<3.5$.
}

$4.5 \times 10^{-13} \mathrm{ph} \mathrm{cm}^{-2} \mathrm{~s}^{-1}$ ) at $\geqslant 1 \mathrm{TeV}$ (Aharonian et al. 2006b; Carrigan et al. 2013). Considering that the Crab Nebula spectrum is harder in the $2 \mathrm{FHL}$ band than in the $>1 \mathrm{TeV}$ band, we estimate that the average sensitivity of $2 \mathrm{FHL}$ in the same region of the H.E.S.S. survey is $\sim 3 \%-4 \%$ of the $50 \mathrm{GeV}-$ $2 \mathrm{TeV}$ Crab Nebula flux. The slightly better sensitivity allows H.E.S.S. to detect 69 sources (as reported in the TeVCat), while the LAT finds 36 objects in the same area. However, the comparable sensitivities of the two surveys allow the study of the properties of the high-energy Galactic population. In the 2FHL catalog there is almost an equal number of SNRs and PWNe in contrast to what is found in the H.E.S.S. survey where the ratio of PWNe to SNRs is 1.5 to 1 . This might be because the hardest PWNe and softest SNRs are difficult to detect, respectively, in the $>50 \mathrm{GeV}$ and $>1 \mathrm{TeV}$ bands.

Of the $362 \mathrm{FHL}$ sources that fall within the footprint of the H.E.S.S. survey, 23 have already been detected at $\mathrm{TeV}$ energies and are associated with known counterparts, while 7 are undetected. The remaining 6 objects (2FHL J1022.0-5750, 2FHL J1505.1-5808，2FHL J1507.4-6213，2FHL J1703.4 -4145 , 2FHL J1745.1-3035 and 2FHL J1856.8+0256) are spatially coincident with $\mathrm{TeV}$ sources whose origin is not known. All of them have hard spectral indices $(\Gamma<2.2)$, but it is interesting to note that 4 of them (2FHL J1022.0-5750, 2FHL J1505.1-5808, 2FHL J1703.4-4145, and 2FHL J1745.1-3035) have $\Gamma<1.7$ (see also Figure 6).

We find that 2FHL J1022.0-5750 is spatially compatible with HESS J1023-575, an extended TeV source (Abramowski et al. 2011c), whose emission might be due to a PWN powered by PSR J1023-5746 (Acero et al. 2013). 2FHL J1505.1 -5808 is spatially coincident with the unidentified object HESS J1503-582, which has a size of 0.26 and a flux above $1 \mathrm{TeV}$ (Renaud et al. 2008) compatible with the extrapolation of the 2FHL J1505.1-5808 spectrum. Its spectrum, reminiscent of that of a PWN (e.g., HESS J1825-137, Grondin et al. 2011) is reported in Figure 6.

2FHL J1507.4-6213 is spatially coincident with HESS J1507-622, an extended source with a radius of 0.15 located 3.5 from the plane (Acero et al. 2011). The analysis of 
Table 2

Description of the Catalog

\begin{tabular}{|c|c|c|c|}
\hline Column & Format & Unit & Description \\
\hline Source_Name & $18 \mathrm{~A}$ & $\cdots$ & 2FHL Source name \\
\hline RAJ2000 & $\mathrm{E}$ & $\operatorname{deg}$ & R.A. \\
\hline DEJ2000 & $\mathrm{E}$ & deg & decl. \\
\hline GLON & $\mathrm{E}$ & deg & Galactic longitude \\
\hline GLAT & $\mathrm{E}$ & $\operatorname{deg}$ & Galactic latitude \\
\hline Pos_Err_68 & $\mathrm{E}$ & deg & Position uncertainty at $68 \%$ confidence level \\
\hline $\mathrm{TS}$ & $\mathrm{E}$ & $\cdots$ & Test Statistic \\
\hline Spectral_Index & $\mathrm{E}$ & $\cdots$ & Observed spectral index \\
\hline Unc_Spectral_Index & $\mathrm{E}$ & $\cdots$ & $1 \sigma$ uncertainty on the observed spectral index \\
\hline Intr_Spectral_Index_D11 & $\mathrm{E}$ & $\cdots$ & Intrinsic spectral index computed using the Domínguez et al. (2011b) EBL model \\
\hline Unc_Intr_Spectral_Index_D11 & $\mathrm{E}$ & $\cdots$ & $\begin{array}{l}1 \sigma \text { Uncertainty on the intrinsic spectral index computed using the Domínguez et al. (2011b) EBL } \\
\text { model }\end{array}$ \\
\hline Intr_Spectral_Index_G12 & $\mathrm{E}$ & $\cdots$ & Intrinsic spectral index computed using the Gilmore et al. (2012) EBL model \\
\hline Unc_Intr_Spectral_Index_G12 & $\mathrm{E}$ & $\cdots$ & $1 \sigma$ Uncertainty on the intrinsic spectral index computed using the Gilmore et al. (2012) EBL model \\
\hline Flux50 & $\mathrm{E}$ & photon $\mathrm{cm}^{-2} \mathrm{~s}^{-1}$ & Integral photon flux from $50 \mathrm{GeV}$ to $2 \mathrm{TeV}$ \\
\hline Unc_Flux50 & $\mathrm{E}$ & photon $\mathrm{cm}^{-2} \mathrm{~s}^{-1}$ & $1 \sigma$ uncertainty on integral photon flux from $50 \mathrm{GeV}$ to $2 \mathrm{TeV}$ \\
\hline Energy_Flux50 & $\mathrm{E}$ & 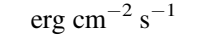 & Energy flux from $50 \mathrm{GeV}$ to $2 \mathrm{TeV}$ \\
\hline Unc_Energy_Flux50 & $\mathrm{E}$ & $\operatorname{erg~} \mathrm{cm}^{-2} \mathrm{~s}^{-1}$ & $1 \sigma$ uncertainty on energy flux from $50 \mathrm{GeV}$ to $2 \mathrm{TeV}$ \\
\hline Flux50_171GeV & $\mathrm{E}$ & photon $\mathrm{cm}^{-2} \mathrm{~s}^{-1}$ & Integral photon flux from 50 to $171 \mathrm{GeV}$ \\
\hline Unc_Flux50_171GeV & $\mathrm{E}$ & photon $\mathrm{cm}^{-2} \mathrm{~s}^{-1}$ & $1 \sigma$ uncertainty on integral photon flux from 50 to $171 \mathrm{GeV}$ \\
\hline Sqrt_TS50_171GeV & $\mathrm{E}$ & photon $\mathrm{cm}^{-2} \mathrm{~s}^{-1}$ & Square root of the Test Statistic between 50 and $171 \mathrm{GeV}$ \\
\hline Flux171_585GeV & $\mathrm{E}$ & photon $\mathrm{cm}^{-2} \mathrm{~s}^{-1}$ & Integral photon flux from 171 to $585 \mathrm{GeV}$ \\
\hline Unc_Flux171_585GeV & $\mathrm{E}$ & photon $\mathrm{cm}^{-2} \mathrm{~s}^{-1}$ & $1 \sigma$ uncertainty on integral photon flux from 171 to $585 \mathrm{GeV}$ \\
\hline Sqrt_TS171_585GeV & $\mathrm{E}$ & photon $\mathrm{cm}^{-2} \mathrm{~s}^{-1}$ & Square root of the Test Statistic between 171 and $585 \mathrm{GeV}$ \\
\hline Flux585_2000GeV & $\mathrm{E}$ & photon $\mathrm{cm}^{-2} \mathrm{~s}^{-1}$ & Integral photon flux from $585 \mathrm{GeV}$ to $2 \mathrm{TeV}$ \\
\hline Unc_Flux585_2000GeV & $\mathrm{E}$ & photon $\mathrm{cm}^{-2} \mathrm{~s}^{-1}$ & $1 \sigma$ uncertainty on integral photon flux from $585 \mathrm{GeV}$ to $2 \mathrm{TeV}$ \\
\hline Sqrt_TS585_2000GeV & $\mathrm{E}$ & photon $\mathrm{cm}^{-2} \mathrm{~s}^{-1}$ & Square root of the Test Statistic between $585 \mathrm{GeV}$ and $2 \mathrm{TeV}$ \\
\hline Npred & $\mathrm{E}$ & $\cdots$ & Predicted number of photons from the source \\
\hline HEP_Energy & $\mathrm{E}$ & $\mathrm{GeV}$ & Highest photon energy \\
\hline HEP_Prob & $\mathrm{E}$ & $\cdots$ & Probability that the HEP is coming from the source, $\geqslant 0.85$ \\
\hline ROI & $\mathrm{E}$ & $\cdots$ & Region of interest number \\
\hline ASSOC & $25 \mathrm{~A}$ & $\cdots$ & Name of the most likely associated source \\
\hline ASSOC_PROB_BAY & $\mathrm{E}$ & $\cdots$ & Probability of association from the Bayesian method \\
\hline ASSOC_PROB_LR & $\mathrm{E}$ & $\cdots$ & Probability of association from the likelihood ratio method \\
\hline CLASS & $8 \mathrm{~A}$ & $\cdots$ & Class designation for the most likely association; see Table 1 \\
\hline Redshift & $\mathrm{E}$ & $\cdots$ & Redshift (when available) of the most likely associated source \\
\hline NuPeak_obs & $\mathrm{E}$ & $\mathrm{Hz}$ & Observed Synchrotron peak frequency \\
\hline 3FGL_Name & $18 \mathrm{~A}$ & $\cdots$ & Name of the most likely associated source in the 3FGL \\
\hline 1FHL_Name & $18 \mathrm{~A}$ & $\cdots$ & Name of the most likely associated source in the 1FHL \\
\hline TeVCat_Name & $18 \mathrm{~A}$ & $\cdots$ & Name of the most likely associated source in the TeVCat \\
\hline
\end{tabular}

Note. A "Source_Name" ending with "e" indicates an extended source.

multiwavelength data showed that it is not possible to discriminate between a hadronic and leptonic origin of the emission, but that the latter scenario, if the emission is powered by a PWN, would require a pulsar generated in the explosion of a hyper-velocity star in order to reach the required distance from the plane (Domainko \& Ohm 2012).

The sources 2FHL J1703.4-4145 and 2FHL J1745.1-3035 are the hardest sources $(\Gamma<1.3)$ among the six objects. 2FHL J1703.4-4145 is spatially coincident with the bright radio emission observed from the western side of the shell of SNR G344.7-001, a nearby mid-aged shell-type (age $\sim 3000$ years and $8^{\prime}$ diameter) SNR (Giacani et al. 2011). Both the 2FHL source and the SNR are spatially coincident with the larger, elongated and unidentified HESS J1702-420 (Aharonian et al. 2008b). It thus seems likely that SNR G344.7-001 is the counterpart of 2FHL J1703.4-4145 and perhaps also of HESS J1702-420. The combined Fermi-H.E.S.S. spectrum of this source is reported in Figure 6.
2FHL J1745.1-3035 is found to be spatially coincident with the extended source HESS J1745-303, which may be comprised of up to three different sources (Aharonian et al. 2008a). Indeed, the position of 2FHL J1745.1-3035 is compatible with the "C" emission region (the second brightest region in the complex, Aharonian et al. 2008a). However, the nature of this source is more complex, because the $2 \mathrm{FHL}$ source is marginally brighter at $1 \mathrm{TeV}$ than the entire H.E.S.S. region and has also a harder spectrum (spectral index of $1.25 \pm 0.38$ in $2 \mathrm{FHL}$ versus $2.17 \pm 0.11$ as measured by H.E.S.S.).

Finally, 2FHL J1856.8+0256 is coincident with HESS $\mathrm{J} 1857+026$, an almost radially symmetric extended source (Aharonian et al. 2008c), whose emission likely originates from a PWN powered by PSR J1856+0245 (Rousseau et al. 2012).

\subsubsection{Extended Source Results}

In total, 31 sources are modeled as spatially extended and input into the ML analysis: 25 listed in 3FGL, 5 sources 
Table 3

Excerpt of the 2FHL Catalog

\begin{tabular}{|c|c|c|c|c|c|c|c|c|c|c|c|c|c|c|c|}
\hline 2FHL Name & R.A. & decl. & $l$ & $b$ & $\theta$ & TS & $F_{50}$ & $\Delta F_{50}$ & $S_{50}$ & $\Delta S_{50}$ & $\Gamma$ & $\Delta \Gamma$ & Association & Class & Redshift \\
\hline $\mathrm{J} 0008.1+4709$ & 2.044 & 47.164 & 115.339 & -15.069 & 0.053 & 28.9 & 1.29 & 0.70 & 1.27 & 0.70 & 6.26 & 2.75 & MG4 J000800+4712 & bll & 2.100 \\
\hline J0009.3+5031 & 2.343 & 50.522 & 116.124 & -11.793 & 0.035 & 54.5 & 2.00 & 0.82 & 2.12 & 0.92 & 5.07 & 1.65 & NVSS J000922+50302 & bll & $\cdots$ \\
\hline J0018.5+2947 & 4.635 & 29.788 & 114.464 & -32.542 & 0.023 & 31.1 & 1.11 & 0.64 & 2.13 & 1.80 & 2.58 & 0.99 & RBS 0042 & bll & 0.100 \\
\hline $\mathrm{J} 0022.0+0006$ & 5.500 & 0.106 & 107.172 & -61.862 & 0.042 & 30.3 & 2.05 & 1.00 & 7.15 & 5.50 & 1.86 & 0.57 & 5BZGJ0022+0006 & bll-g & 0.306 \\
\hline J0033.6-1921 & 8.411 & -19.358 & 94.280 & -81.222 & 0.019 & 149.5 & 5.71 & 1.57 & 7.98 & 2.81 & 3.32 & 0.68 & KUV 00311-1938 & bll & 0.610 \\
\hline J0035.8+5949 & 8.966 & 59.831 & 120.974 & -2.981 & 0.012 & 380.4 & 12.30 & 1.92 & 30.30 & 7.27 & 2.23 & 0.22 & 1ES $0033+595$ & bll & $\cdots$ \\
\hline J0040.3+4049 & 10.095 & 40.832 & 120.676 & -21.992 & 0.020 & 27.0 & 1.10 & 0.66 & 2.97 & 2.79 & 2.12 & 0.81 & B3 $0037+405$ & bcu I & $\cdots$ \\
\hline J0043.9+3424 & 10.976 & 34.411 & 121.164 & -28.435 & 0.051 & 39.9 & 1.91 & 0.86 & 2.13 & 1.04 & 4.56 & 1.60 & GB6 J0043+3426 & fsrq & 0.966 \\
\hline $\mathrm{J} 0045.2+2126$ & 11.319 & 21.445 & 121.020 & -41.404 & 0.036 & 81.9 & 3.51 & 1.19 & 4.82 & 1.98 & 3.38 & 0.82 & GB6 J0045+2127 & bll & $\cdots$ \\
\hline $\mathrm{J} 0048.0+5449$ & 12.002 & 54.828 & 122.433 & -8.040 & 0.049 & 35.4 & 1.27 & 0.64 & 7.99 & 5.97 & 1.30 & 0.51 & 1RXS J004754.5+544 & bcu II & $\ldots$ \\
\hline
\end{tabular}

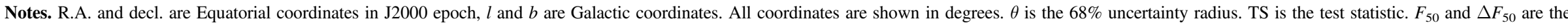

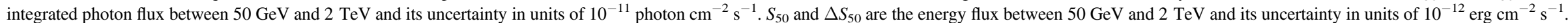

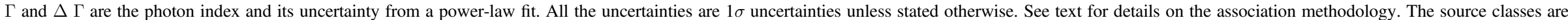
detailed in Table 1. Redshifts were taken from from Shaw et al. (2012, 2013), Masetti et al. (2013) and the NED and SIMBAD databases.

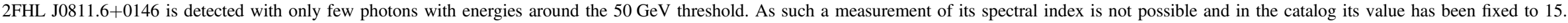

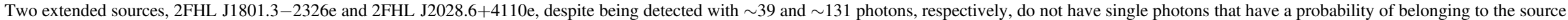
$>85 \%$. As such their "HEP_Energy" and "HEP_Prob" columns are left blank.

(This table is available in its entirety in FITS format.) 


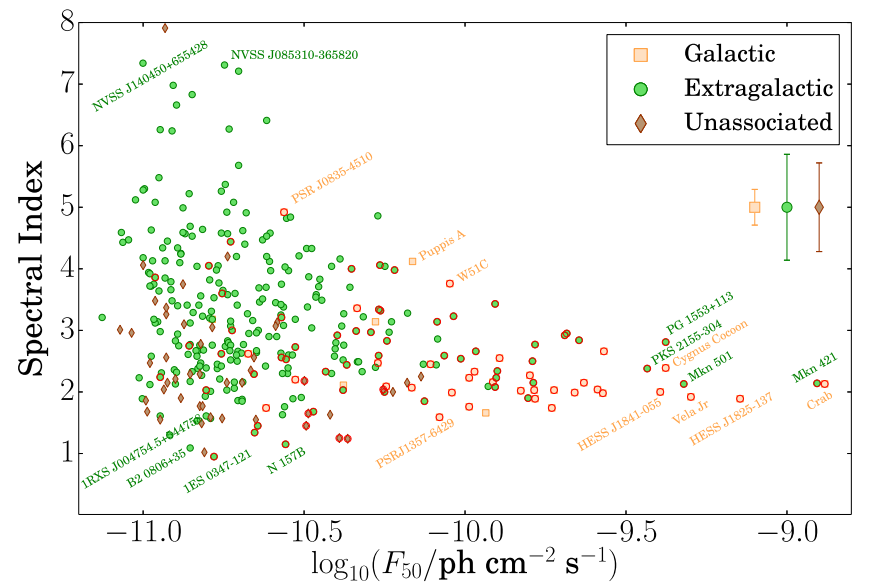

Figure 3. The photon flux vs. the spectral index of Galactic sources (orange squares), extragalactic sources (green circles), and unassociated sources (brown diamonds). The medians of the uncertainties are shown as well. We note that the detectability does not significantly depend on the spectral index as a consequence of the low intensity of the diffuse background and constant PSF over the energy range of the analysis. Symbols with a red outline are sources already detected at TeV energies and contained in the TeVCat catalog.

detected in the pointlike analysis (described in Section 2.3.1) that were not detected as extended at the time of 3FGL, and one, SNR W41, reported recently by both the H.E. S.S. and LAT teams (Abramowski et al. 2015a). Names and properties of the extended sources are provided in Tables 4 and 5. Six extended sources, detected in 3FGL, were not detected in 2FHL: the SMC, S 147 (the point source 2FHL J0534.1+2753 was detected inside it), the lobes of Centaurus A (although we detect its core as a point source, 2FHL J1325.6-4301), W 44, HB 21 and the Cygnus Loop.

We detect a weak source, 2FHL J1714.1-4012 (TS = 27), just outside the southwestern edge of the 3FGL spatial template used to model the emission from SNR RX J1713.7-3946 (2FHL J1713.5-3945e). 2FHL J1714.1-4012 has a hard spectral index $\Gamma=1.63 \pm 0.38$, that is within errors of the spectral index derived for the $\mathrm{SNR}, \Gamma=2.03 \pm 0.20$. It is unclear whether 2FHL J1714.1-4012 is a distinct source separated from the SNR, or the result of un-modeled residual emission due to an imperfection in the spatial template adopted for the extended source.

2FHL J1836.5-0655e is associated with the PWN HESS J1837-069. The 3FGL catalog contains several point sources in the vicinity of the PWN. We detect three sources in the vicinity, 2FHL J1834.5-0701, 2FHL J1837.4-0717, and 2FHL J1839.5-0705, the first two of which are coincident with 3FGL sources (3FGL J1834.6-0659, 3FGL J1837.6 -0717 , respectively). The power-law spectral indices of the three 2FHL point sources and 2FHL J1836.5-0655e are all consistent with each other. The concentration of sources around HESS J1837-069 combined with the spectral compatibility of the sources is suggestive of a common origin to the $\gamma$-ray emission in this region. However, the surrounding $\gamma$ rays could arise from other sources in the region (Gotthelf \& Halpern 2008); further analysis is necessary to determine the nature of the sources in this region.

A brief description of the five new 2 FHL extended sources is given below with residual TS maps for the region surrounding each source shown in Figure 7. Detailed analyses of these new extended sources will be reported in separate papers.

2FHL J1443.2-6221e overlaps with the young, radiodetected SNR RCW 86 (G315.42.3). RCW 86 is a $42^{\prime}$ diameter SNR that lies at a distance of $2.3-2.8 \mathrm{kpc}$ and is likely associated with the first recorded supernova, SN 185 AD (Rosado et al. 1996; Sollerman et al. 2003). With more than 40 months of data and using the P7SOURCE data set, the LAT did not significantly detect the SNR, but upper limits on detection at $\mathrm{GeV}$ energies combined with detection of significant extension in the $\mathrm{TeV}$ (Aharonian et al. 2009) were sufficient to strongly favor a leptonic origin for the emission (LemoineGoumard et al. 2012).

An updated LAT analysis of RCW 86 using 76 months of data, as well as the Pass 8 event-level analysis, resulted in detection of the SNR by the LAT as well as significant extension measurement (Hewitt \& Fermi-LAT Collaboration 2015). In this paper, we report the results derived for 2FHL J1443.2-6221e from the pointlike analysis described in Section 2.3.1.

2FHL J1419.2-6048e is a newly detected extended sources with size $\sigma_{\text {disk }}=0^{\circ} .36 \pm 0.03$, that overlaps two nearby PWN/ PSR complexes in the Kookaburra region. In the southwest of Kookaburra, HESS J1418-609 (Aharonian et al. 2006a) is coincident with both the extended non-thermal X-ray "Rabbit" PWN (G313.3+0.1, Roberts et al. 1999), and the $\gamma$-ray detected pulsar PSR J1418-6058 (Abdo et al. 2009). The northeast region, called "K3," contains HESS J1420-607, coincident with PWN G313.5+0.3 and PSR J1420-6048. Acero et al. (2013) detected, with Fermi-LAT, emission from
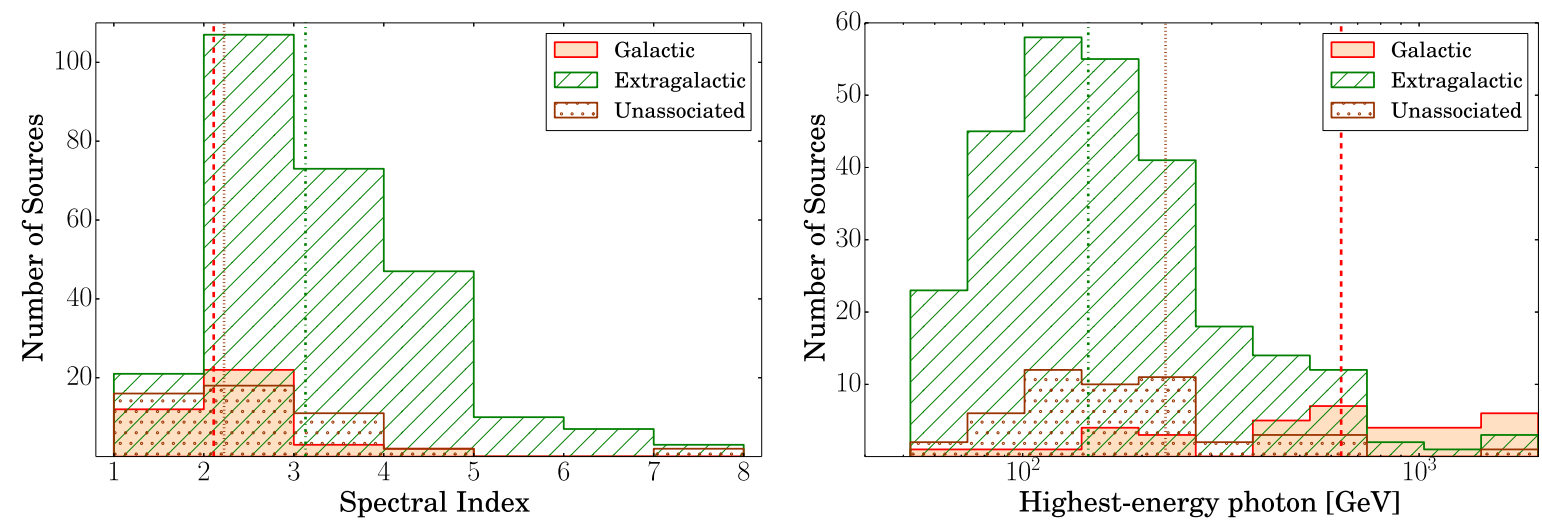

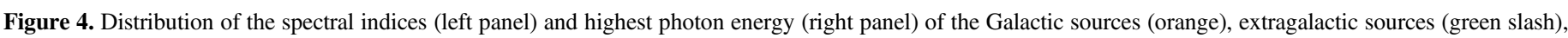

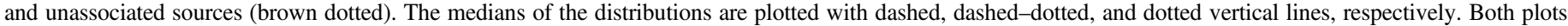
show that a distinct population of hard-spectrum sources is of Galactic origin. 

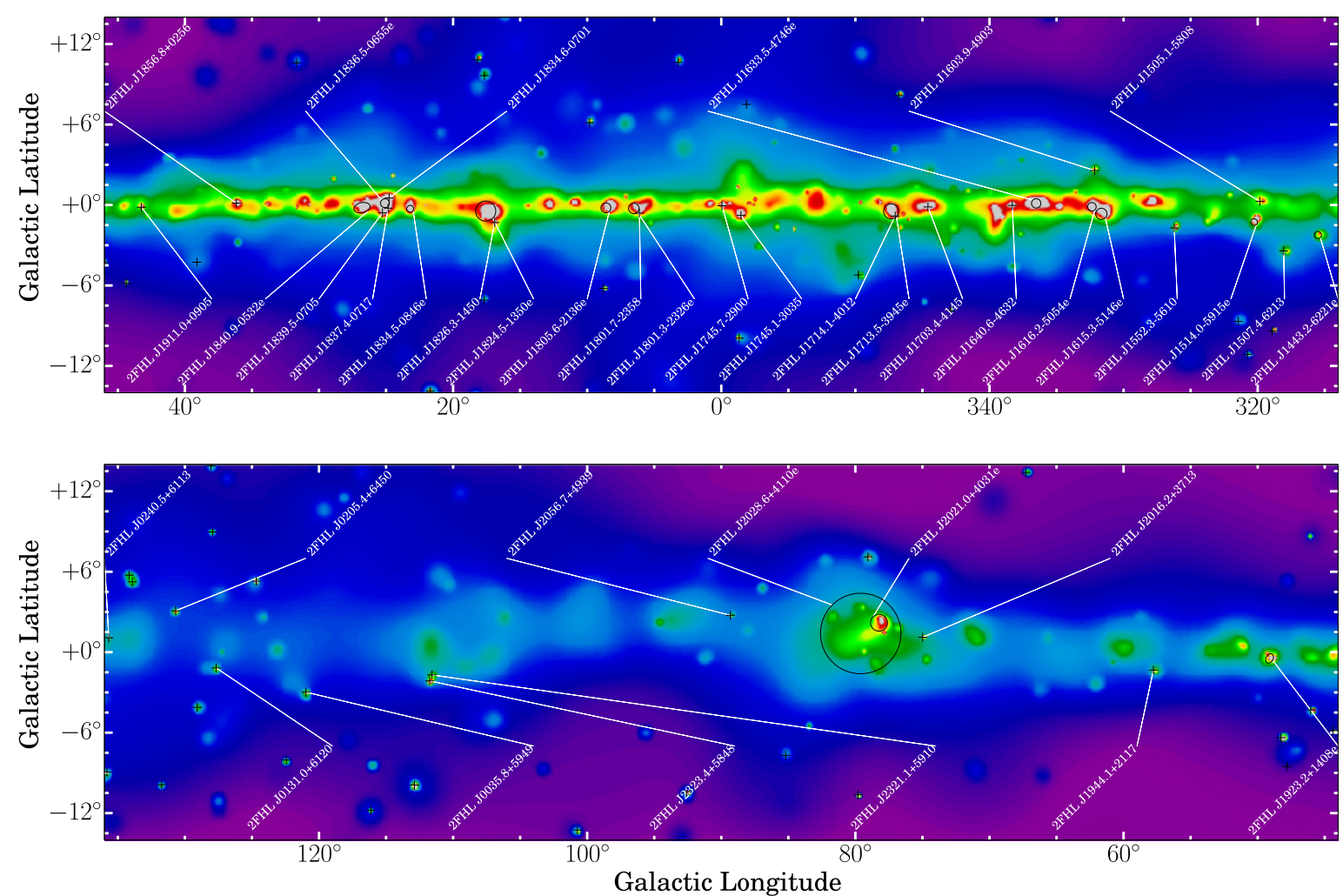

Figure 5. Adaptively smoothed count map showing the whole Galactic plane $0^{\circ} \leqslant l \leqslant 360^{\circ}$ at Galactic latitudes $-14^{\circ} \leqslant b \leqslant 14^{\circ}$ divided in four panels. The panels are centered at $l=0^{\circ}, 90^{\circ}, 180^{\circ}$, and $270^{\circ}$, respectively. Detected point sources are marked with a cross whereas extended sources are indicated with their extensions. Only sources located at $-4^{\circ} \leqslant b \leqslant 4^{\circ}$ are explicitly named, plus the Crab Nebula.
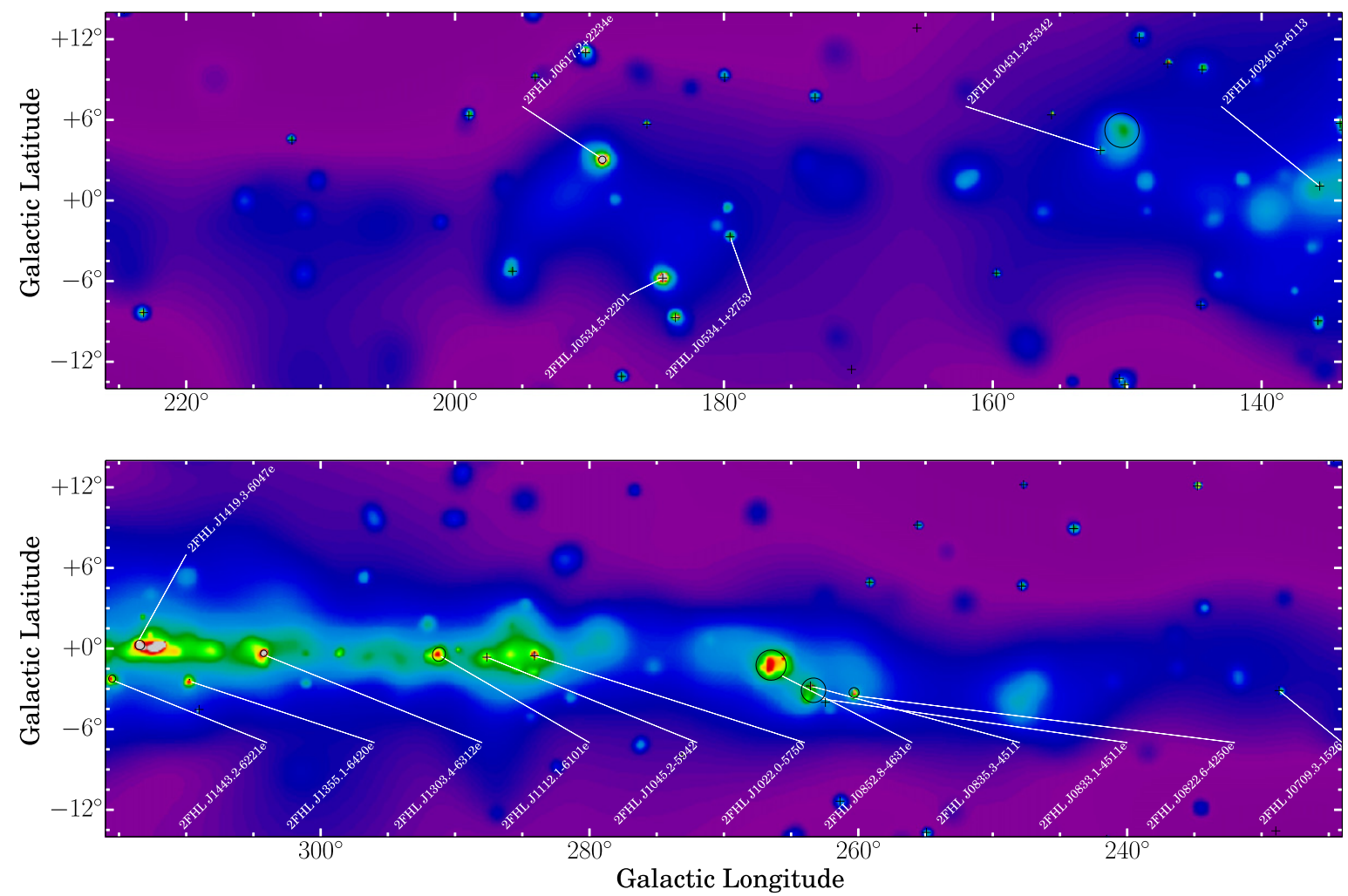

Figure 5. (Continued.) 

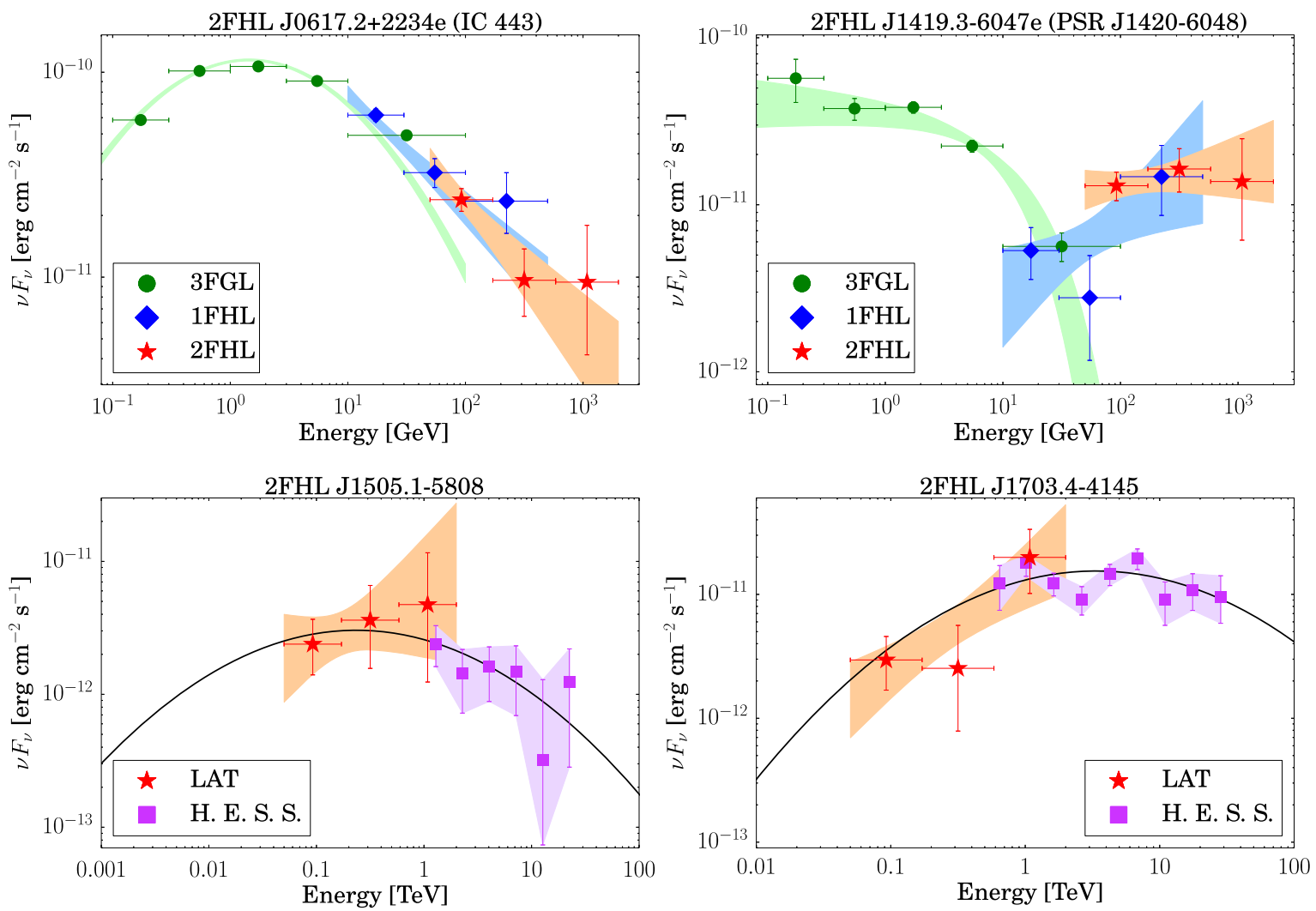

Figure 6. Spectral energy distributions of four Galactic sources constructed by combining data from the 3FGL (green diamonds), 1FHL (blue circles), and 2FHL (red stars). We show the 3FGL extended source SNR IC 443 (top left), the new 2FHL extended source PSR J1420-6048 (top right), and two "dark accelerators" detected by H.E.S.S. at TeV energies (Carrigan et al. 2013, purple squares) without a previous LAT counterpart: HESS J1503-582 (bottom left) and HESS J1702-420 (bottom right).

both HESS J1418-609 (with a soft spectral index, pulsar-like spectrum) and HESS J1420-607 (with a hard power-law index) above $10 \mathrm{GeV}$, but only HESS J1420-607 was significantly detected above $30 \mathrm{GeV}$. Neither showed significant extension. Our result for the fitted power-law spectral index of 2FHL J1419.2-6048e is in agreement with the previous $\mathrm{GeV}$ and $\mathrm{TeV}$ results, yet our measured radius is considerably larger than the $\mathrm{TeV}$ extension. To compare the extensions of the uniform disk model used for 2FHL J1419.2 $-6048 \mathrm{e}$ in this paper to the Gaussian model of Aharonian et al. (2006a), we defined the radius which contains 68\% of the source's intensity as $r_{68}$, with $r_{68 \text {,Gaussian }}=1.51 \sigma$, and $r_{68}$, disk $=0.82 \sigma$ (Lande et al. 2012). We find that

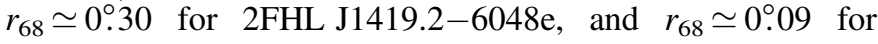
HESS J1420-607.

2FHL J1355.2-6430e, coincident with the VHE source HESS J1356-645, is detected as extended $\left(\sigma_{\text {disk }}=\right.$ $0.57 \pm 0.02)$ for the first time by the LAT in this work. The source HESS J1356-645 (Abramowski et al. 2011a) is associated with the pulsar PSR J1357-6429, which was determined to be powering a surrounding extended radio and X-ray PWN (Lemoine-Goumard et al. 2011). Acero et al. (2013) detected faint emission from the nebula, and derived a $99 \%$ c.l. Bayesian upper limit on extension $\left(\sigma_{\text {Gauss }}<0.39\right)$ in the absence of significant extension. The fitted spectral index for 2FHL J1355.2-6430e is compatible with the $\mathrm{GeV}$ and $\mathrm{TeV}$ results (Acero et al. 2013; Abramowski et al. 2011a), however, the fitted disk extension is larger than that of the $\mathrm{TeV}$ detection, with $r_{68} \simeq 0^{\circ} .47$ for $2 \mathrm{FHL}$ J1355.2-6430e and $r_{68} \simeq 0^{\circ} .30$ for HESS J1356-645.
2FHL J1112.4-6059e is an extended source $\left(\sigma_{\text {disk }}=\right.$ $0.53 \pm 0.03$ ) newly detected by the LAT that encircles two 3FGL sources, 3FGL J1111.9-6058 and 3FGL J1111.96038, and has another, 3FGL J1112.0-6135, just outside its boundary (Acero et al. 2015). The extended source also partially overlaps the massive star-forming region NGC 3603.

Finally, 2FHL J0431.2+5553e is a large extended source $\left(\sigma_{\text {disk }}=1^{\circ} .27 \pm 0.04\right)$, with a hard spectrum, that has not been previously detected at $\gamma$-ray energies. It overlaps the recently discovered radio SNR G150.3+4.5 (Gao \& Han 2014). G150.3 +4.5 is a $2^{\circ} .5 \times 3^{\circ}$ (Galactic coordinates) elliptical shell type SNR that has a steep radio synchrotron spectrum $(\alpha=-0.6)$, indicative of radio SNRs.

\subsection{The 2FHL Extragalactic Sky}

\subsubsection{General and Spectral Properties}

Most of the sources detected in 2FHL are extragalactic. 83\% (299) are either located at $|b| \geqslant 10^{\circ}$ or associated with an extragalactic source. We refer to this set of sources as the extragalactic sample.

BL Lacs represent the most numerous source class (54\% of the full 2FHL catalog and $65 \%$ of the extragalactic sample), while there are a few detected FSRQs (10 sources, 3\% of the full 2FHL catalog). Such a low number of FSRQs is expected due to their soft spectra at $>50 \mathrm{GeV}$. Most of the detected BL Lacs belong to the high-frequency synchrotron peak (HSP) class, rather than to the low-frequency synchrotron peak (LSP), or intermediate-frequency synchrotron peak (ISP) class. This is 
Table 4

2FHL Extended Sources Previously Detected by the Fermi-LAT

\begin{tabular}{|c|c|c|c|c|c|c|c|}
\hline 2FHL Name & $l(\mathrm{deg})$ & $b(\mathrm{deg})$ & TS & Association & Class & Spatial model & Extension (deg) \\
\hline J0526.6-6825e & 278.843 & -32.850 & 49.80 & LMC & gal & 2D Gaussian & 1.87 \\
\hline $\mathrm{J} 0617.2+2234 \mathrm{e}$ & 189.048 & 3.033 & 398.64 & IC 443 & snr & 2D Gaussian & 0.27 \\
\hline J0822.6-4250e & 260.317 & -3.277 & 63.87 & Puppis A & snr & Disk & 0.37 \\
\hline J0833.1-4511e & 263.333 & -3.104 & 49.70 & Vela X & pwn & Disk & 0.91 \\
\hline J0852.8-4631e & 266.491 & -1.233 & 437.21 & Vela Jr & snr & Disk & 1.12 \\
\hline $\mathrm{J} 1303.4-6312 \mathrm{e}$ & 304.235 & -0.358 & 56.06 & HESS J1303-631 & pwn & 2D Gaussian & 0.24 \\
\hline J1514.0-5915e & 320.269 & -1.276 & 165.51 & MSH 15-52 & pwn & Disk & 0.25 \\
\hline $\mathrm{J} 1615.3-5146 \mathrm{e}$ & 331.659 & -0.659 & 128.15 & HESS J1614-518 & spp & Disk & 0.42 \\
\hline $\mathrm{J} 1616.2-5054 \mathrm{e}$ & 332.365 & -0.131 & 87.18 & HESS J1616-508 & pwn & Disk & 0.32 \\
\hline $\mathrm{J} 1633.5-4746 \mathrm{e}$ & 336.517 & 0.121 & 114.17 & HESS J1632-478 & pwn & Disk & 0.35 \\
\hline $\mathrm{J} 1713.5-3945 \mathrm{e}$ & 347.336 & -0.473 & 60.98 & RX J1713.7-3946 & snr & Map & 0.56 \\
\hline $\mathrm{J} 1801.3-2326 \mathrm{e}$ & 6.527 & -0.251 & 50.20 & W 28 & snr & Disk & 0.39 \\
\hline $\mathrm{J} 1805.6-2136 \mathrm{e}$ & 8.606 & -0.211 & 160.43 & W 30 & snr & Disk & 0.37 \\
\hline $\mathrm{J} 1824.5-1350 \mathrm{e}$ & 17.569 & -0.452 & 266.09 & HESS J1825-137 & pwn & 2D Gaussian & 0.75 \\
\hline $\mathrm{J} 1834.9-0848 \mathrm{e}$ & 23.216 & -0.373 & 67.30 & W 41 & snr & 2D Gaussian & 0.23 \\
\hline $\mathrm{J} 1836.5-0655 \mathrm{e}$ & 25.081 & 0.136 & 62.72 & HESS J1837-069 & pwn & Disk & 0.33 \\
\hline $\mathrm{J} 1840.9-0532 \mathrm{e}$ & 26.796 & -0.198 & 163.15 & HESS J1841-055 & pwn & Elliptical & $0.62,0.38,39.0$ \\
\hline $\mathrm{J} 1923.2+1408 \mathrm{e}$ & 49.112 & -0.466 & 44.60 & W 51C & snr & Elliptical & $0.38,0.26,90.0$ \\
\hline $\mathrm{J} 2021.0+4031 \mathrm{e}$ & 78.241 & 2.197 & 115.97 & Gamma Cygni & snr & Disk & 0.63 \\
\hline $\mathrm{J} 2028.6+4110 \mathrm{e}$ & 79.601 & 1.396 & 28.09 & Cygnus Cocoon & sfr & 2D Gaussian & 3.0 \\
\hline
\end{tabular}

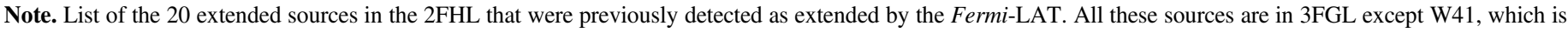

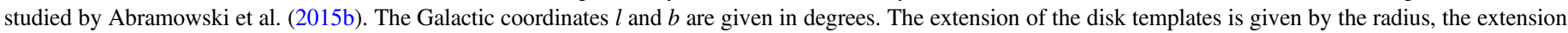

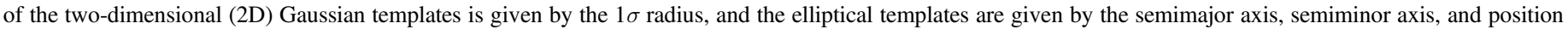
angle (east of north).

Table 5

New 2FHL Extended Sources

\begin{tabular}{|c|c|c|c|c|c|c|c|c|c|c|c|c|}
\hline 2FHL Name & $l(\mathrm{deg})$ & $b(\mathrm{deg})$ & TS & $\mathrm{TS}_{\text {ext }}$ & $\mathrm{TS}_{2 \mathrm{pts}}$ & $F_{50}$ & $\Delta F_{50}$ & $\Gamma$ & $\Delta \Gamma$ & Association & Class & Radius (deg) \\
\hline $\mathrm{J} 0431.2+5553 \mathrm{e}$ & 150.384 & 5.216 & 87.9 & 83.4 & 26.2 & 11.70 & 2.11 & 1.66 & 0.20 & G $150.3+4.5$ & snr & 1.27 \\
\hline $\mathrm{J} 1112.4-6059 \mathrm{e}$ & 291.222 & -0.388 & 80.9 & 68.3 & 22.5 & 12.80 & 2.36 & 2.15 & 0.28 & PSR J1112-6103 & pwn & 0.53 \\
\hline $\mathrm{J} 1355.2-6430 \mathrm{e}$ & 309.730 & -2.484 & 82.3 & 31.8 & 12.9 & 9.59 & 1.95 & 1.56 & 0.22 & PSR J1357-6429 & pwn & 0.57 \\
\hline $\mathrm{J} 1419.2-6048 \mathrm{e}$ & 313.432 & 0.260 & 109.3 & 49.1 & 15.6 & 17.60 & 2.80 & 1.87 & 0.19 & PSR J1420-6048 & pwn & 0.36 \\
\hline $\mathrm{J} 1443.2-6221 \mathrm{e}$ & 315.505 & -2.239 & 75.6 & 29.9 & 19.2 & 7.23 & 1.70 & 2.07 & 0.30 & SNR G315.4-2.3 & snr & 0.27 \\
\hline
\end{tabular}

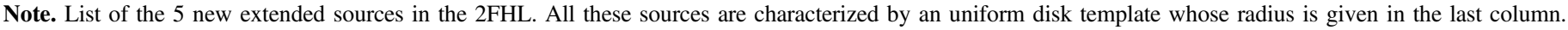

shown in Figure 8, which reports the distribution of synchrotron peak frequencies of blazars detected in the 2FHL and in the 3FGL. It is clear that the two catalogs sample different parts of the blazar population, with the 3FGL including mostly LSPs and ISPs and the 2FHL including mostly HSPs.

There are 198 2FHL sources in the extragalactic sample that are detected both in the 3FGL and 1FHL catalogs. There are 33 other sources that are neither in the 3FGL nor in the 1FHL catalog (and not in 1FGL/2FGL either). In general, 2FHL sources not detected in 3FGL and 1FHL are harder (median $\Gamma \sim 2.8$ versus 3.2), fainter (median $F_{50} \sim$ $1.3 \times 10^{-11} \mathrm{ph} \mathrm{cm}^{-2} \mathrm{~s}^{-1}$ versus $\left.2.3 \times 10^{-11} \mathrm{ph} \mathrm{cm}^{-2} \mathrm{~s}^{-1}\right)$ and are detected at lower significance (median TS $\sim 30$ versus 53 ) than those detected in the aforementioned catalogs.

One of the 33 exclusive 2FHL sources is 2FHL J1944.1 $+2117(\Gamma=2.73 \pm 0.66)$ that is associated with HESS J1943 +213 (Abramowski et al. 2011b). This source is potentially an extreme high-frequency peaked BL Lac, classified as bcu II, located in the Galactic plane with $\log _{10}\left(\nu_{\text {peak }}^{S} / \mathrm{Hz}\right)=18.79$ (Ackermann et al. 2015). We also detect N 157B, 2FHL
J0537.4-6908, with a very hard spectral index of $\Gamma=1.15 \pm 0.37$. This is an extragalactic PWN in the LMC detected by H.E.S.S. up to $18 \mathrm{TeV}$ (Abramowski et al. 2012, 2015c). The other 31 sources (out of the 33 2FHL exclusive sources) are not detected by Cherenkov telescopes yet. Of the 31 sources, 16 are classified as some type of blazar whereas 14 are unclassified. One source is associated with a galaxy cluster, 2FHL J0318.0-4414 detected with a soft spectrum $(\Gamma=4.0 \pm 1.7)$. The emission likely originates from PKS $0316-444$, which is a bright radio source at the center of Abell $3112(z=0.075$, Takizawa et al. 2003). Emission from the region of Abell 3112 was already marginally found by Ackermann et al. (2014a), yet it is robustly detected in the 2FHL.

We find that the number of FSRQs is strongly reduced from 1FHL (71 sources) to the 2FHL (10 sources). This is mainly due to the soft indices of this blazar population and the higher energy threshold adopted in the 2FHL with respect to the 1FHL. Another indication of this effect is that only 2 sources at $z>1$ (out of a total of 7) are FSRQs, while the rest are BL Lacs. Other noteworthy examples of detected extragalactic 

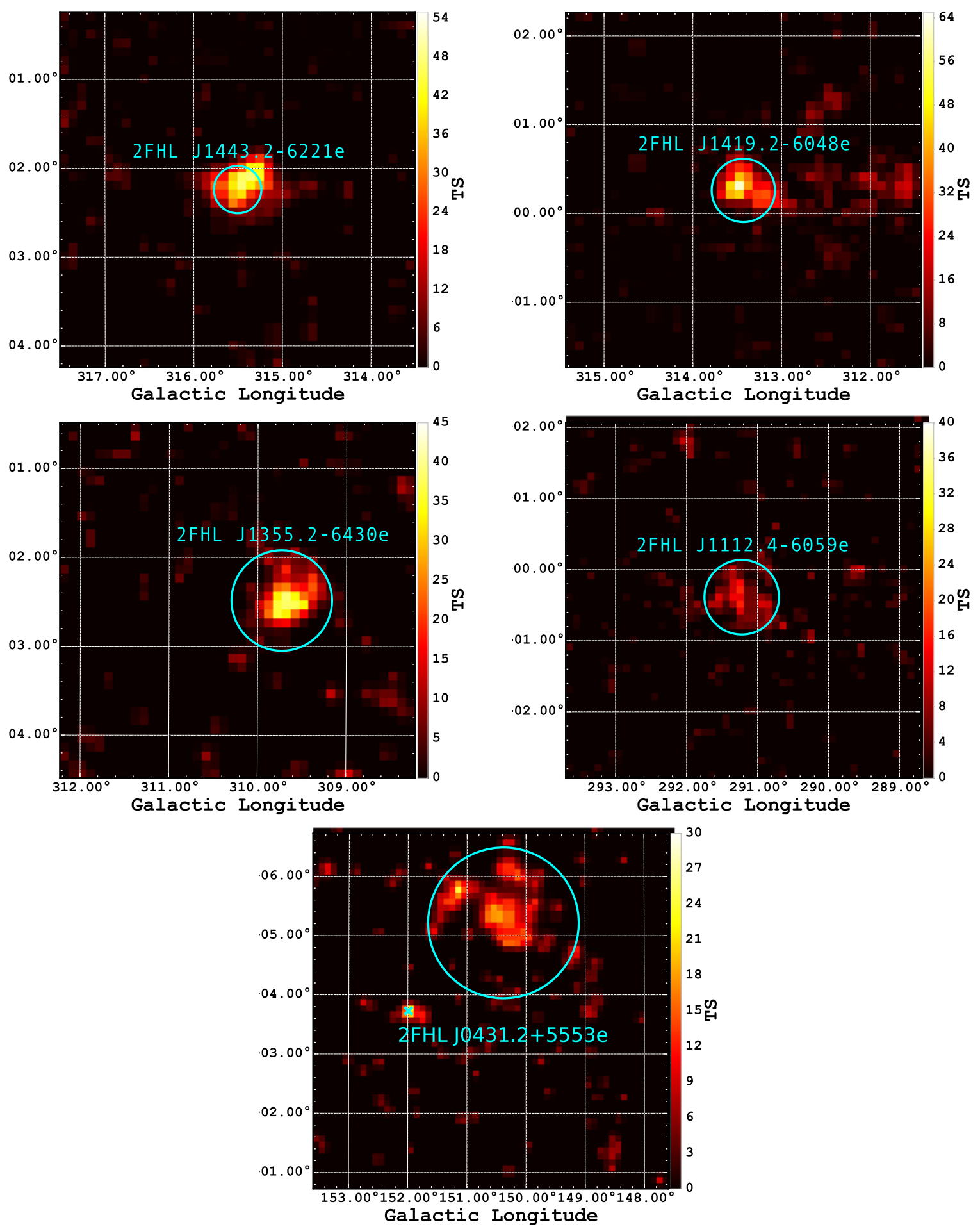

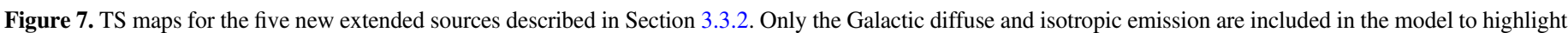

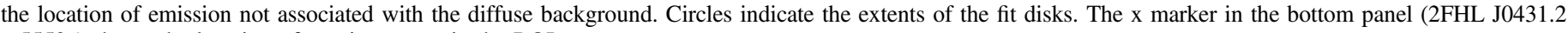
$+5553 \mathrm{e}$ ) shows the location of a point source in the ROI.

sources are the nearby radio galaxies IC $310(z=0.019)$, NGC $1275(z=0.0175)$, PKS 0625-35 $(z=0.05494), 3 \mathrm{C} 264$ $(z=0.021718)$, M $87 \quad(z=0.004283)$, and Centaurus A $(z=0.0018)$.

Figure 9 shows the SEDs of two notable sources, Mrk 421 and $3 \mathrm{C}$ 66A, highlighting how the LAT now resolves the descending part of the high-energy peak. Indeed, 2FHL spectra of extragalactic sources are generally softer than the corresponding 3FGL and 1FHL spectra. This is evident in Figure 10, which compares the spectral indices for a subsample of $158 \mathrm{BL}$ Lacs in common between the $2 \mathrm{FHL}$, 3FGL and 1FHL catalogs. There are two interesting facts illustrated by Figure 10. First, the median of the distribution shifts toward larger (softer) indices since 2FHL samples the drop of the SED. Second, the scatter of the distribution becomes larger with increasing energies. Some of the scatter 


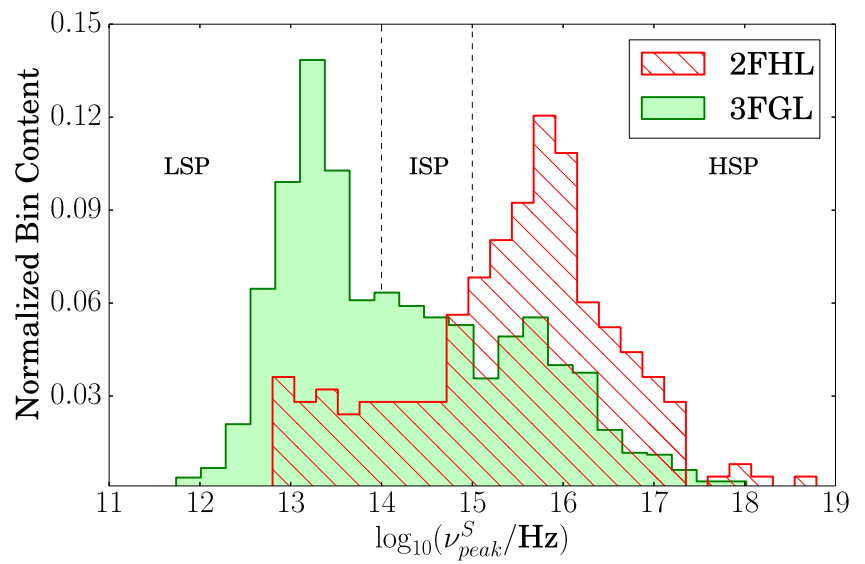

Figure 8. Normalized distribution of the frequency of the synchrotron peak for the blazars detected in the 3FGL and those detected in the 2FHL. For the 3FGL sources the peak frequencies were adopted from 3LAC (Ackermann et al. 2015). LSP, ISP, and HSP blazars are those with $\log _{10}\left(\nu_{\text {peak }}^{S} / \mathrm{Hz}\right)<14$, $14<\log _{10}\left(\nu_{\text {peak }}^{S} / \mathrm{Hz}\right)<15$, and $\log _{10}\left(\nu_{\text {peak }}^{S} / \mathrm{Hz}\right)>15$, respectively.

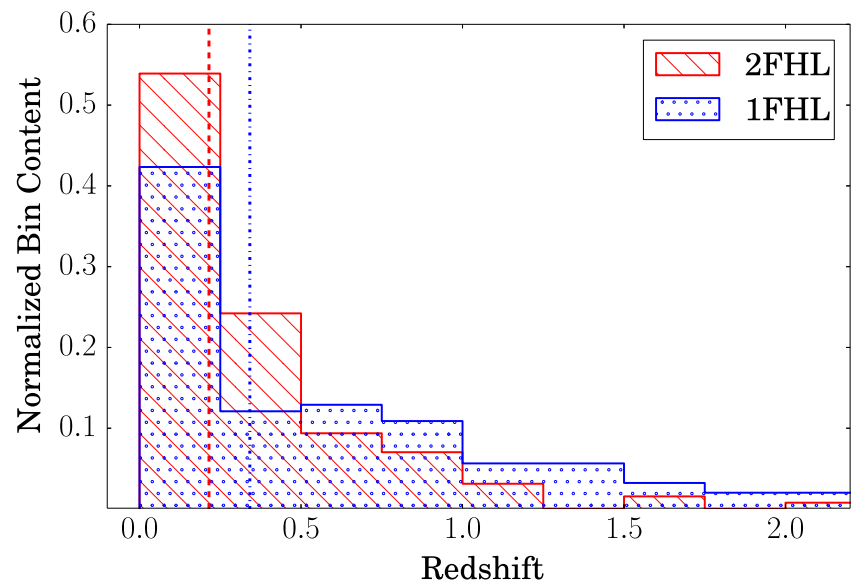

Figure 11. Normalized redshift distribution of the sources with known redshift in 2FHL (orange backslash) and 1FHL (blue dotted). The medians of the distributions are plotted with a dashed and dashed-dotted vertical line, respectively.
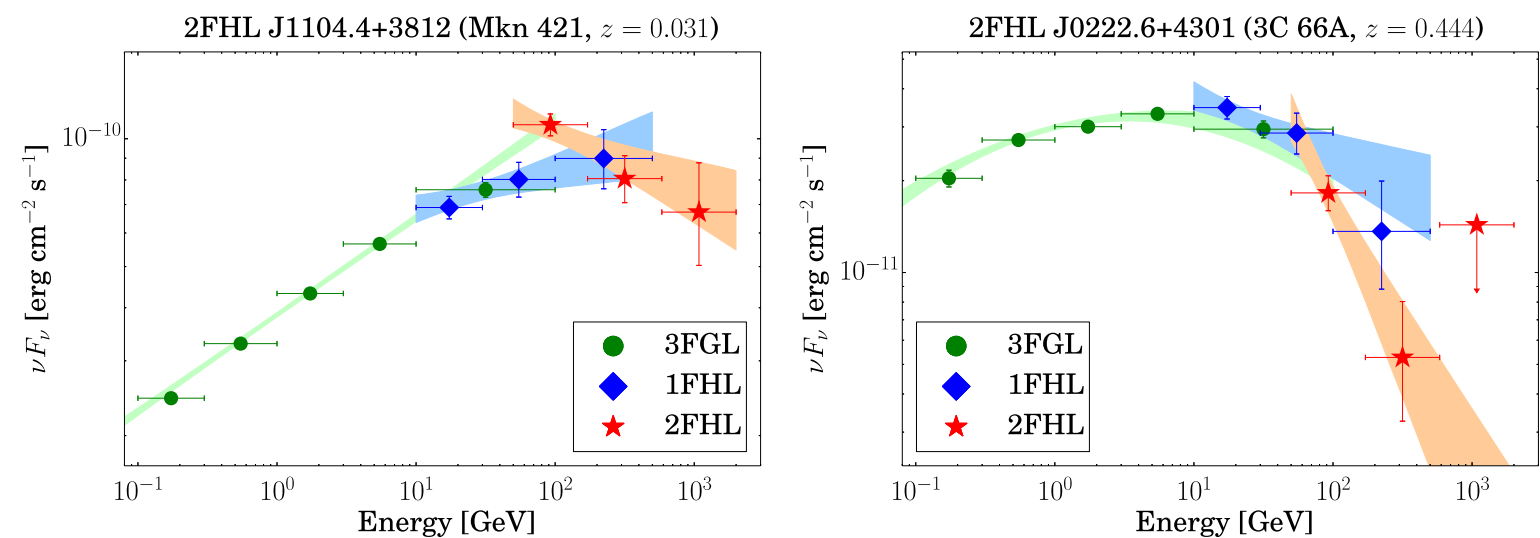

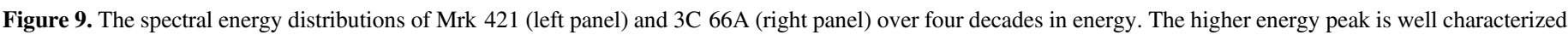
by combining data from the 3FGL (green diamonds), 1FHL (blue circles), and 2FHL (red stars).

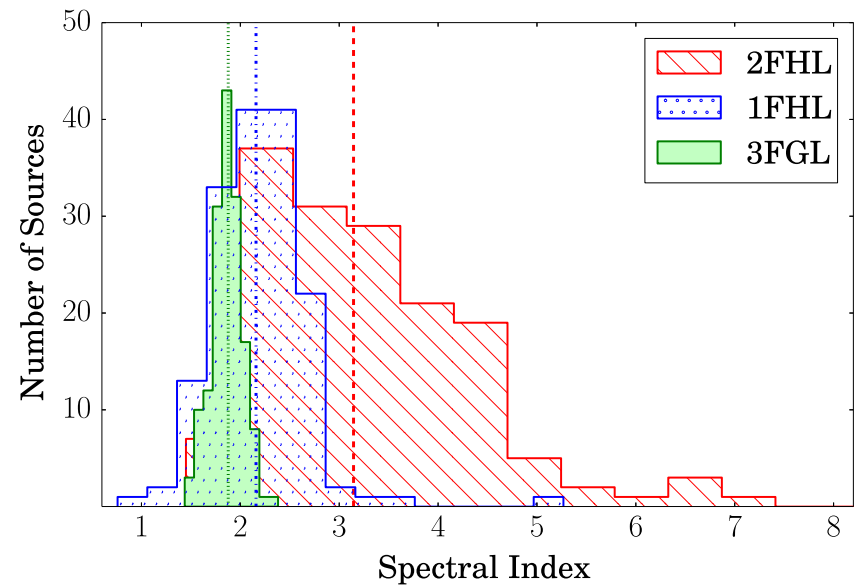

Figure 10. The distribution of spectral indices for a subsample of $158 \mathrm{BL}$ Lacs that are in common among the 2FHL (backslash orange), 3FGL (slash green), and 1FHL (purple). The medians of the distributions are shown with vertical lines. The higher the energy band, the larger the index; therefore sources get softer with increasing energy. The scatter of the distribution is also larger with increasing energy, partly because of the lower statistics.

arises from statistical effects since the number of detected photons at $>50 \mathrm{GeV}$ is generally lower than in the 3FGL and 1FHL.

\subsection{2. $\gamma$-ray Attenuation}

Spectroscopic redshift measurements exists in the literature (e.g., Shaw et al. 2012, 2013; Masetti et al. 2013) for $43 \%$ (128) of the 299 extragalactic sources. The comparison of the 2FHL and 1FHL redshift distributions (reported in Figure 11) shows that on average $2 \mathrm{FHL}$ sources lie at lower redshifts than the $1 \mathrm{FHL}$ ones. This might be because FSRQs and LSP blazars, which tend to be located at higher redshifts, have soft spectra and are faint in the 2FHL band. High-redshift sources appear even fainter because of the EBL attenuation. The EBL contains all the photons, from the ultraviolet to the infrared, that have been emitted by star formation processes and supermassive black hole accretion throughout the history of the universe (e.g., Dwek \& Krennrich 2013). The spectrum of extragalactic $\gamma$-ray sources, in the whole energy range of our analysis, is modified by pair-production interaction of the $\gamma$-ray photons with the EBL (e.g., Ackermann et al. 2012). This interaction produces an attenuation of the observed fluxes that is energy and redshift dependent (the larger the $\gamma$-ray energy and/or the redshift, the larger the attenuation). We note that despite the high energies probed by this work, seven sources have $z>1$, where the EBL attenuation is not negligible. These sources are associated with PKS 0454-234 $(z=1.003)$, PKS 0426-380 ( $z=1.105$, Tanaka et al. 2013), OJ $014(z=1.148)$, TXS 0628 


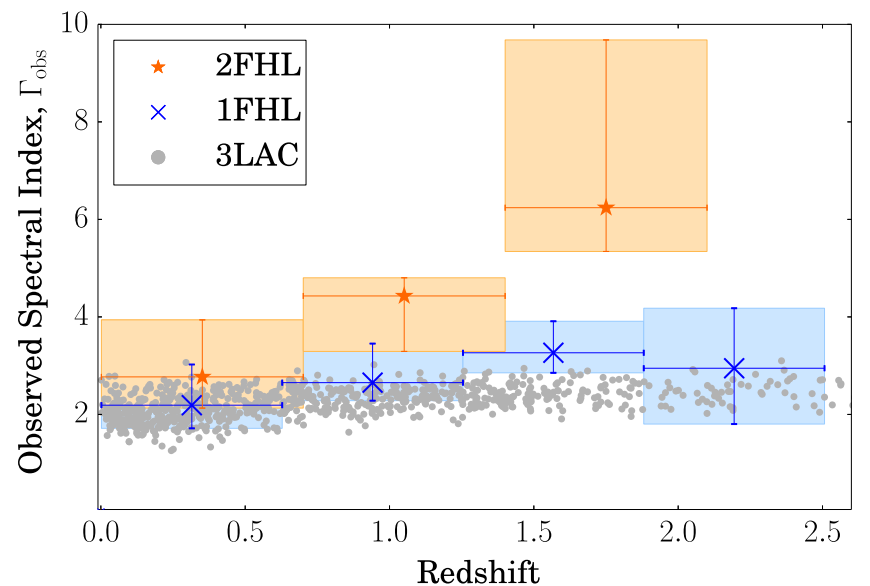

Figure 12. Observed spectral index vs. redshift of the 3LAC sources (energy range, $0.1-100 \mathrm{GeV}$ ), the median spectral index of the 1FHL sources $(10-500 \mathrm{GeV})$ in 4 redshift bins, and the median spectral index of the 2FHL sources $(50-2 \mathrm{TeV})$ in 3 redshift bins. The uncertainties are calculated as the $68 \%$ containment around the median. There is a dependence of the spectral index on redshift at the energies where the EBL attenuation is significant.

$-240(z=1.238)$, PKS B1424-418 $(z=1.55)$, B2 $2114+33$ $(z=1.6)$, and MG4 J000800+4712 $(z=2.1)$. We specially note that three sources have redshifts $z>1.5$. The effect of the EBL attenuation is clearly seen in Figure 12. This figure shows the significant dependence of the spectral index on the redshift at the 2FHL energies whereas that dependence is reduced at lower energies (i.e., for 3FGL and 1FHL) where the EBL interaction is less relevant.

The EBL attenuation can be parameterized by an optical depth $\tau$ that can be derived from empirical EBL models (e.g., Franceschini et al. 2008; Finke et al. 2010; Kneiske \& Dole 2010; Domínguez et al. 2011b; Gilmore et al. 2012; Helgason \& Kashlinsky 2012; Stecker et al. 2012; Scully et al. 2014; Khaire \& Srianand 2014). In fact, the EBL sets a distance limit from where $\gamma$-ray photons of a given energy are expected to reach us, the cosmic $\gamma$-ray horizon (CGRH, e.g., Abdo et al. 2010; Domínguez et al. 2013). Formally, the CGRH may be defined as the energy at which $\tau=1$ as a function of redshift. Figure 13 shows the HEPs in 2 FHL versus the redshift of emission. As we can see from Figure 13, a few photons are from near and beyond the CGRH. These photons from significantly attenuated sources provide information about the EBL, which in turn carries fundamental information about galaxy evolution and cosmology (e.g., Hauser \& Dwek 2001; Domínguez \& Prada 2013; Biteau \& Williams 2015). In fact, the most complete EBL information is typically given by the sources detected up to the highest energies, which are usually the brightest sources with the hardest spectra. The HEPs with the largest optical depths are associated with RBS 0413 $(z=0.19, \tau=1.38)$, PKS 0823-223 $(z=0.911, \tau=1.39)$, RX J0648.7+1516 $(z=0.179, \quad \tau=1.74), \quad$ 1ES 0347-121 $(z=0.188, \quad \tau=2.43), \quad$ and $1 \mathrm{ES} 0502+675 \quad(z=0.34$, $\tau=2.84$ ), where $\tau$ is given by the observationally based EBL model by Domínguez et al. (2011b). These photons are especially interesting for testing alternative photon-propagation scenarios such as secondary cascades (Essey \& Kusenko 2010) and axion-like particles (e.g., de Angelis et al. 2007; SánchezConde et al. 2009; Domínguez et al. 2011a; Horns \& Meyer 2012). However, we caution the reader that Figure 13 cannot be readily interpreted in terms of constraints on the EBL

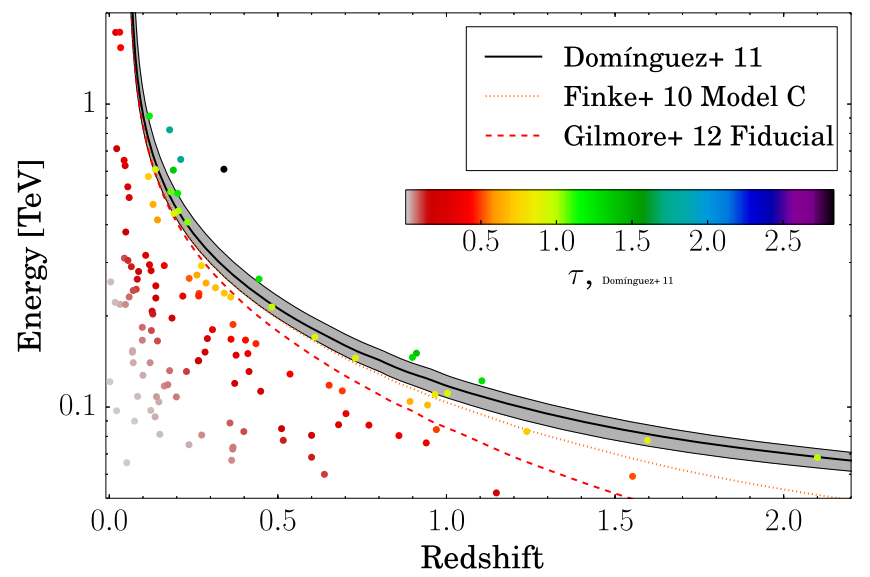

Figure 13. The highest photon energy vs. source redshift. The symbols are color coded by the optical depth, $\tau$, estimated from the EBL model by Domínguez et al. (2011b). Different estimates of the cosmic $\gamma$-ray horizon are plotted as well, which are derived from the EBL models by Finke et al. (2010, dotted orange line), Domínguez et al. (2011b, solid black line, with its uncertainties as a shaded band) and Gilmore et al. (2012, dashed red line). We note that several photons are from near and beyond the horizon.

optical depth because brighter and harder sources stand a larger chance to have photons detected from beyond the horizon.

\subsection{Variability of $2 F H L$ Sources}

We have studied the variability of $2 \mathrm{FHL}$ sources using the same Bayesian Block algorithm adopted in 1FHL (Ackermann et al. 2013). This algorithm, designed to detect and characterize variability in time series (Scargle 1998; Scargle et al. 2013), is well suited in regimes of low-count statistics. The algorithm partitions a given source light curve into constant segments (blocks), each characterized by a flux and duration. The location of the transition between blocks is determined by optimizing a fitness function (using the algorithm of Jackson et al. 2005) for the partitions. As for the analysis of 1FHL data, the fitness function employed here is the logarithm of the ML for each individual block under the hypothesis of a constant local flux (see Scargle et al. 2013). A 1\% false positive threshold was selected for all sources. For 360 sources, we expect 3-4 false detections. Aperture photometry was performed for each source using an ROI of 0.5 radius centered on the ML source coordinates. The light curves were divided into 50 equal time bins spanning the 80 -month interval. For sources with neighboring $2 \mathrm{FHL}$ sources closer than $1^{\circ}$, the radius of the ROI was decreased to the greater value of (half the angular separation) or 0.25 . No background subtraction was done for the aperture photometry and Bayesian Block analyses. This is the same procedure that was used in 1FHL. Five pairs of sources are closer than 0.5 , all of which are located in the Galactic plane.

Only 7 sources are detected as being variable at more than 99\% C.L (see Table 6) in contrast to the detection of 43 variable sources in 1FHL. Most of the $2 \mathrm{FHL}$ variable sources are associated with BL Lacs and all but one (PMN J1603 -4904 , associated with an AGN that may be a young radio galaxy, Müller et al. 2015) have already been detected by IACTs. Most sources are detected with only 2 blocks, meaning that they were brighter during part of the 80 months spanned by the 2FHL analysis. MG4 J200112+4352, 1ES 0502+675, 
Table 6

Results of the Bayesian Block Variability Analysis

\begin{tabular}{|c|c|c|c|c|}
\hline 2FHL Name & Number of Blocks & $\gamma$-ray Association & Class & TeV? \\
\hline J0035.8+5949 & 2 & $1 \mathrm{ES} 0033+595$ & bll & Yes \\
\hline $\mathrm{J} 0222.6+4301$ & 3 & $3 \mathrm{C} 66 \mathrm{~A}$ & bll & Yes \\
\hline J0319.7+1849 & 2 & RBS 0413 & bll & Yes \\
\hline J0507.9+6737 & 2 & 1ES $0502+675$ & bll & Yes \\
\hline $\mathrm{J} 1104.4+3812$ & 4 & Mrk 421 & bll & Yes \\
\hline $\mathrm{J} 1603.9-4903$ & 2 & PMN J1603-4904 & agn & No \\
\hline $\mathrm{J} 2001.2+4352$ & 2 & MG4 J200112+4352 & bll & Yes \\
\hline
\end{tabular}

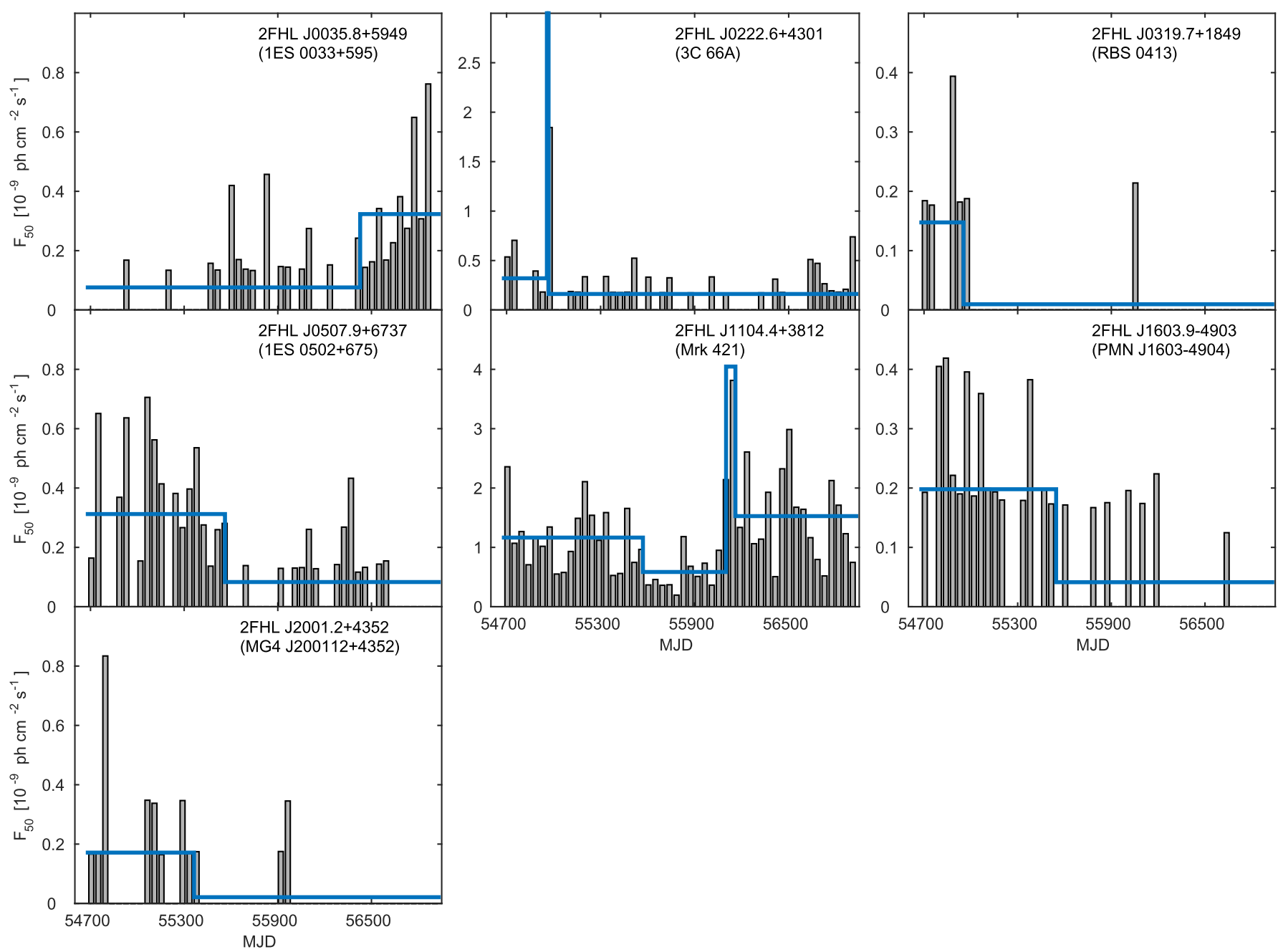

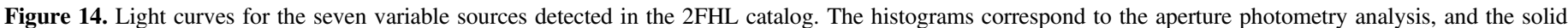

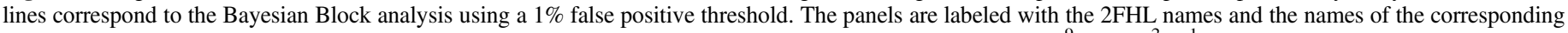

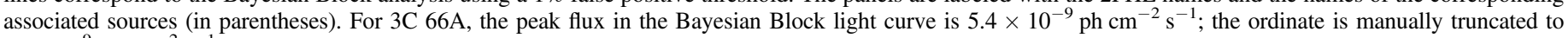
$3 \times 10^{-9} \mathrm{ph} \mathrm{cm}^{-2} \mathrm{~s}^{-1}$ to improve readability.

RBS 0413, and PMN J1603-4904 were all in high states at the beginning of the Fermi mission, while 1ES $0033+595$ became brighter after 2013. 3C 66A had a clear outburst at $>50 \mathrm{GeV}$, coincident with the bright flare detected by VERITAS and Fermi in 2008 September (Abdo et al. 2011). The most frequently variable source (detected with 4 blocks) is Mrk 421, which was not detected as variable in 1FHL. The Bayesian Block analysis clearly detects the outburst of the source in 2012 August (Hovatta et al. 2015). Figure 14 shows the light curves for these 7 sources. Half of the sources in 2FHL are detected with less than 6 photons, which prevents us from assessing whether variability is present. Indeed, the weakest source for which we detect variability is 2FHL J0319.7+1849 (RBS 0413), which is detected with $\sim 6$ photons.

\subsection{Candidates for Detection with Cherenkov Telescopes}

A cross correlation between the 2FHL and TeVCat catalogs, finds that 282 sources in 2FHL (i.e., $~ 78 \%$ of the $2 \mathrm{FHL}$ sample) have not been detected yet by IACTs. Figure 15 shows the photon flux distributions of the population already detected by IACTs (median of $5.72 \times 10^{-10} \mathrm{ph} \mathrm{cm}^{-2} \mathrm{~s}^{-1}$ ) and that not 


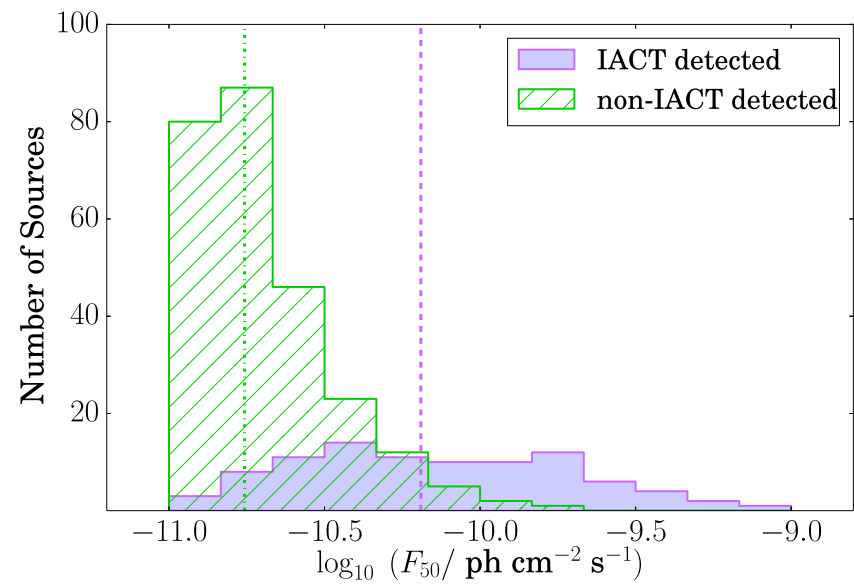

Figure 15. Photon flux distributions of the 2FHL population that has been detected already by IACTs (purple) and that has not yet been detected (green). The medians of the distributions are shown with vertical lines.

detected (median of $1.55 \times 10^{-10} \mathrm{ph} \mathrm{cm}^{-2} \mathrm{~s}^{-1}$ ); the flux is a factor $\sim 4$ greater for the population already detected. Out of 282 sources that are not detected by IACTs, 216 are located at $|b| \geqslant 10^{\circ}$ and 66 are at $|b|<10^{\circ}$. Therefore, most of the 2FHL targets for IACTs are likely extragalactic. Given the energy threshold and sensitivity of current IACTs, a large majority of sources in our catalog are promising targets for IACT detections. However, for extragalactic studies, it may be convenient to concentrate on HSP blazars, which typically have their higher-energy peak at energies above $100 \mathrm{GeV}$. With a factor of 10 improvement in sensitivity and the extension to energies below $100 \mathrm{GeV}$, all 2FHL sources should be detectable by the future Cherenkov Telescope Array (Acharya et al. 2013).

\section{SUMMARY}

We have presented an all-sky analysis at $\geqslant 50 \mathrm{GeV}$ of 80 months of Fermi-LAT data relying on the new Pass 8 eventlevel analysis. Pass 8 delivers improvements in the acceptance and the PSF, reduces background of misclassified charged particles and extends the energy range at which Fermi-LAT is sensitive. All this allowed Fermi-LAT to detect 360 sources in the $50 \mathrm{GeV}-2 \mathrm{TeV}$ range, performing an unbiased census of the $>50 \mathrm{GeV}$ sky for the first time. This catalog of sources (dubbed 2FHL) provides a bridge between the traditional $0.1-100 \mathrm{GeV}$ band of Fermi-LAT catalogs (Acero et al. 2015) and the $\gtrsim 100 \mathrm{GeV}$ band probed by IACTs from the ground. The $2 \mathrm{FHL}$ catalog has the potential to improve the efficiency with which new sources are detected at $\mathrm{TeV}$ energies since only about $25 \%$ of the 2FHL sources were previously detected by IACTs.

The majority $(\gtrsim 80 \%)$ of sources detected in the $2 \mathrm{FHL}$ catalog are likely extragalactic because they are either located at high Galactic latitude or are associated with blazars. BL Lacs represent the largest population of sources detected by the LAT (54\%), followed by blazars of uncertain classification $(16 \%)$ and unassociated sources $(13 \%)$. Most BL Lacs in 2FHL belong to the HSP class and display the hardest (among blazars) $\gamma$-ray spectra and substantial emission in the $50 \mathrm{GeV}-$ $2 \mathrm{TeV}$ band. These sources are powerful probes of the EBL and this work has shown that Fermi-LAT has detected emission from many blazars at optical depths $>1$.
The 2FHL includes 103 sources in the direction of the Galactic plane $\left(|b|<10^{\circ}\right)$. While a fraction of the sources $(\sim 39 \%)$ is associated with blazars, the rest are Galactic and unassociated sources. Galactic sources generally display much harder photon indices than blazars (median of $\sim 2$ versus $\sim 3$ ) and copious $\mathrm{TeV}$ emission, both signs of efficient particle acceleration. Most Galactic sources are associated with PWNe and SNRs, systems at the end of the stellar evolution cycle, and are detected as spatially extended. All the hard (spectral index $<2$ ) unassociated sources within the plane of our Galaxy are likely of Galactic origin, since very few blazars have spectra as hard.

A comparison with a similarly long data set of Pass 7 photons shows that Pass 8 allows the LAT to detect $35 \%$ more sources. It is thus clear that Pass 8 and the accumulated exposure allow the LAT to extend its reach to higher energy and to open a new window on the sub-TeV sky. Sensitivity improves linearly with time in the photon-limited regime, thus further observations by the LAT in the coming years will probe the $>50 \mathrm{GeV}$ sky even more deeply, providing important targets for current and future Cherenkov telescopes.

The Fermi LAT Collaboration acknowledges generous ongoing support from a number of agencies and institutes that have supported both the development and the operation of the LAT as well as scientific data analysis. These include the National Aeronautics and Space Administration and the Department of Energy in the United States, the Commissariat à l'Energie Atomique and the Centre National de la Recherche Scientifique/Institut National de Physique Nucléaire et de Physique des Particules in France, the Agenzia Spaziale Italiana and the Istituto Nazionale di Fisica Nucleare in Italy, the Ministry of Education, Culture, Sports, Science and Technology (MEXT), High Energy Accelerator Research Organization (KEK) and Japan Aerospace Exploration Agency (JAXA) in Japan, and the K. A. Wallenberg Foundation, the Swedish Research Council and the Swedish National Space Board in Sweden. Additional support for science analysis during the operations phase is gratefully acknowledged from the Istituto Nazionale di Astrofisica in Italy and the Centre National d'Études Spatiales in France. This research has made use of the NASA/IPAC Extragalactic Database (NED) which is operated by the Jet Propulsion Laboratory, California Institute of Technology, under contract with the National Aeronautics and Space Administration. This research has made use of the SIMBAD database, operated at CDS, Strasbourg, France.

Facility: Fermi/LAT.

\section{REFERENCES}

Abdo, A. A., Ackermann, M., Ajello, M., et al. 2009, Sci, 325, 840 Abdo, A. A., Ackermann, M., Ajello, M., et al. 2010, ApJ, 723, 1082 Abdo, A. A., Ackermann, M., Ajello, M., et al. 2011, ApJ, 726, 43 Abdo, A. A., Ajello, M., Allafort, A., et al. 2013, ApJS, 208, 17 Abramowski, A., Acero, F., Aharonian, F., et al. 2011a, A\&A, 533, A103 Abramowski, A., Acero, F., Aharonian, F., et al. 2011b, A\&A, 529, A49 Abramowski, A., Acero, F., Aharonian, F., et al. 2011c, A\&A, 525, A46 Abramowski, A., Acero, F., Aharonian, F., et al. 2012, A\&A, 545, L2 Abramowski, A., Aharonian, F., Ait Benkhali, F., et al. 2015a, A\&A, 574, A27 Abramowski, A., Aharonian, F., Ait Benkhali, F., et al. 2015b, A\&A, 574, A27 Abramowski, A., Aharonian, F., Ait Benkhali, F., et al. 2015c, Sci, 347, 406 Acero, F., Aharonian, F., Akhperjanian, A. G., et al. 2011, A\&A, 525, A45 Acero, F., Ackermann, M., Ajello, M., et al. 2013, ApJ, 773, 77 Acero, F., Ackermann, M., Ajello, M., et al. 2015a, arXiv:1501.02003 
Acero, F., Ackermann, M., Ajello, M., et al. 2015b, arXiv:1511.06778

Acharya, B. S., Actis, M., Aghajani, T., et al. 2013, APh, 43, 3

Ackermann, M., Ajello, M., Allafort, A., et al. 2012, Sci, 338, 1190

Ackermann, M., Ajello, M., Allafort, A., et al. 2013, ApJS, 209, 34

Ackermann, M., Ajello, M., Albert, A., et al. 2014a, ApJ, 787, 18

Ackermann, M., Albert, A., Atwood, W. B., et al. 2014b, ApJ, 793, 64

Ackermann, M., Ajello, M., Atwood, W., et al. 2015, 3LAC paper, arXiv: 1501.06054

Aharonian, F., Akhperjanian, A. G., Bazer-Bachi, A. R., et al. 2006a, A\&A, 456,245

Aharonian, F., Akhperjanian, A. G., Bazer-Bachi, A. R., et al. 2006b, ApJ, 636, 777

Aharonian, F., Akhperjanian, A. G., Barres de Almeida, U., et al. 2008a, A\&A, 483, 509

Aharonian, F., Akhperjanian, A. G., Barres de Almeida, U., et al. 2008b, A\&A, 477,353

Aharonian, F., Akhperjanian, A. G., Barres de Almeida, U., et al. 2008c, A\&A, 477, 353

Aharonian, F., Akhperjanian, A. G., de Almeida, U. B., et al. 2009, ApJ, 692,1500

Atwood, W. B., Abdo, A. A., Ackermann, M., et al. 2009, ApJ, 697, 1071

Atwood, W. B., Baldini, L., Bregeon, J., et al. 2013a, ApJ, 774, 76

Atwood, W. B., Albert, A., Baldini, L., et al. 2013b, arXiv:1303.3514

Biteau, J., \& Williams, D. A. 2015, arXiv:1502.04166

Carrigan, S., Brun, F., Chaves, R. C. G., et al. 2013, arXiv:1307.4868

Ciprini, S., Tosti, G., Marcucci, F., et al. 2007, in AIP Conf. Ser. 921, The First GLAST Symposium, ed. S. Ritz, P. Michelson \& C. A. Meegan (Melville, NY: AIP), 546

Condon, J. J., Cotton, W. D., Greisen, E. W., et al. 1998, AJ, 115, 1693

de Angelis, A., Roncadelli, M., \& Mansutti, O. 2007, PhRvD, 76, 121301

Domainko, W., \& Ohm, S. 2012, A\&A, 545, A94

Domínguez, P., \& Ajello, M. 2015, ApJL, 813, L34

Domínguez, A., Finke, J. D., Prada, F., et al. 2013, ApJ, 770, 77

Domínguez, A., \& Prada, F. 2013, ApJL, 771, L34

Domínguez, A., Sánchez-Conde, M. A., \& Prada, F. 2011a, JCAP, 11, 20

Domínguez, A., Primack, J. R., Rosario, D. J., et al. 2011b, MNRAS, 410, 2556

Dwek, E., \& Krennrich, F. 2013, APh, 43, 112

Essey, W., \& Kusenko, A. 2010, APh, 33, 81

Finke, J. D., Razzaque, S., \& Dermer, C. D. 2010, ApJ, 712, 238

Franceschini, A., Rodighiero, G., \& Vaccari, M. 2008, A\&A, 487, 837

Gao, X. Y., \& Han, J. L. 2014, A\&A, 567, A59

Giacani, E., Smith, M. J. S., Dubner, G., \& Loiseau, N. 2011, A\&A, 531, A138

Gilmore, R. C., Somerville, R. S., Primack, J. R., \& Domínguez, A. 2012, MNRAS, 422, 3189

Gotthelf, E. V., \& Halpern, J. P. 2008, ApJ, 681, 515

Grondin, M.-H., Funk, S., Lemoine-Goumard, M., et al. 2011, ApJ, 738, 42

Hauser, M. G., \& Dwek, E. 2001, ARA\&A, 39, 249

Helgason, K., \& Kashlinsky, A. 2012, ApJL, 758, L13
Hewitt, J. W., \& Fermi-LAT Collaboration 2015, in American Astronomical Society Meeting Abstracts, 225, 140.31

Hinton, J. 2004, NewAR, 48, 331

Holder, J., Acciari, V., Aliu, E., et al. 2009, in AIP Conf. Proc. 1085 (Melville, NY: AIP), 657

Horns, D., \& Meyer, M. 2012, JCAP, 2, 33

Hovatta, T., Petropoulou, M., Richards, J. L., et al. 2015, MNRAS, 448, 3121

Jackson, B., Scargle, J. D., Barnes, D., et al. 2005, ISPL, 12, 105

Kerr, M. 2010, PhD thesis, Univ. Washington, arXiv:1101.6072

Khaire, V., \& Srianand, R. 2014, arXiv:1405.7038

Kneiske, T. M., \& Dole, H. 2010, A\&A, 515, A19

Lande, J., Ackermann, M., Allafort, A., et al. 2012, ApJ, 756, 5

Lemoine-Goumard, M., Renaud, M., Vink, J., et al. 2012, A\&A, 545, A28

Lemoine-Goumard, M., Zavlin, V. E., Grondin, M.-H., et al. 2011, A\&A, 533, A102

Lorenz, E. 2004, NewAR, 48, 339

Manchester, R. N., Hobbs, G. B., Teoh, A., \& Hobbs, M. 2005, AJ, 129 1993

Masetti, N., Sbarufatti, B., Parisi, P., et al. 2013, arXiv:1310.1916

Massaro, E., Maselli, A., Leto, C., Marchegiani, P., Perri, M., Giommi, P., \& Piranomonte, S. 2015, arXiv:1502.07755

Mauch, T., Murphy, T., Buttery, H. J., et al. 2003, MNRAS, 342, 1117

Müller, C., Krauß, F., Dauser, T., et al. 2015, A\&A, 574, A117

Nolan, P. L., Abdo, A. A., Ackermann, M., et al. 2012, ApJS, 199, 31

Ong, R. A. 2013, arXiv:1307.5003

Renaud, M., Goret, P., \& Chaves, R. C. G. 2008, in AIP Conf. Ser. 1085, ed. F. A. Aharonian, W. Hofmann \& F. Rieger (Melville, NY: AIP), 281

Roberts, M. S. E., Romani, R. W., Johnston, S., \& Green, A. J. 1999, ApJ, 515,712

Rosado, M., Ambrocio-Cruz, P., Le Coarer, E., \& Marcelin, M. 1996, A\&A, 315,243

Rousseau, R., Grondin, M.-H., Van Etten, A., et al. 2012, A\&A, 544, A3

Sánchez-Conde, M. A., Paneque, D., Bloom, E., Prada, F., \& Domínguez, A. 2009, PhRvD, 79, 123511

Scargle, J. D. 1998, ApJ, 504, 405

Scargle, J. D., Norris, J. P., Jackson, B., \& Chiang, J. 2013, ApJ, 764, 167

Scully, S. T., Malkan, M. A., \& Stecker, F. W. 2014, ApJ, 784, 138

Shaw, M. S., Romani, R. W., Cotter, G., et al. 2012, ApJ, 748, 49

Shaw, M. S., Romani, R. W., Cotter, G., et al. 2013, ApJ, 764, 135

Sollerman, J., Ghavamian, P., Lundqvist, P., \& Smith, R. C. 2003, A\&A, 407, 249

Stecker, F. W., Malkan, M. A., \& Scully, S. T. 2012, ApJ, 761, 128

Su, M., Slatyer, T. R., \& Finkbeiner, D. P. 2010, ApJ, 724, 1044

Takizawa, M., Sarazin, C. L., Blanton, E. L., \& Taylor, G. B. 2003, ApJ, 595,142

Tanaka, Y. T., Cheung, C. C., Inoue, Y., et al. 2013, ApJL, 777, L18

Voges, W., Aschenbach, B., Boller, T., et al. 1999, A\&A, 349, 389

Voges, W., Aschenbach, B., Boller, T., et al. 2000, yCat, 9029, 0 